\title{
Adaptive Neural Control with Prespecified Tracking Accuracy for a Class of Switched Systems Subject to Input Delay
}

\author{
Xu Zhang, ${ }^{1}$ Jian $W u\left(\mathbb{D},{ }^{2} \mathrm{Wu} \mathrm{Ai}^{3}\right.$ and Jing $\mathrm{Li}^{4}$ \\ ${ }^{1}$ School of Mathematics and Computation Science, Anqing Normal University, Anqing 246133, China \\ ${ }^{2}$ The University Key Laboratory of Intelligent Perception and Computing of Anhui Province, Anqing Normal University, \\ Anqing 246133, China \\ ${ }^{3}$ College of Science, Guilin University of Technology, Guilin, China \\ ${ }^{4}$ School of Mathematics and Statistics, Xidian University, Xian 710071, China
}

Correspondence should be addressed to Jian Wu; jwu2011@126.com

Received 21 January 2019; Revised 8 June 2019; Accepted 27 June 2019; Published 9 July 2019

Academic Editor: Toshikazu Kuniya

Copyright (c) 2019 Xu Zhang et al. This is an open access article distributed under the Creative Commons Attribution License, which permits unrestricted use, distribution, and reproduction in any medium, provided the original work is properly cited.

\begin{abstract}
This paper is concerned with the adaptive tracking control design for a class of uncertain switched systems subject to input delay. Unlike the existing results on uncertain switched systems, the new proposed control scheme ensures that the tracking error converges to the accuracy given a priori according to the requirement. To achieve this aim, some nonnegative switching functions are introduced to replace the conventional Lyapunov function. In addition, neural networks are used to approximate the unknown simultaneous domination functions. By combining the backstepping technique and some common nonnegative switching functions, a stable adaptive neural controller is established. It can be shown that the closed-loop system is semiglobally uniformly ultimately bounded (SGUUB) and the tracking error satisfies the predefined accuracy. The effectiveness of the proposed control scheme is verified by a simulation example.
\end{abstract}

\section{Introduction}

As we all know, switched systems are a special class of hybrid systems, which have been paid much attention due to their practical applications. Many practical engineering systems are well described by switched systems such as networked system, robotic system, and traffic surveillance and control system $[1,2]$. It provides a strong motivation for investigating the switched systems. Over the past two decades, a lot of scholars focused on dealing with the control issues of the switched systems, and a number of interesting and meaningful results have been presented in [3-10]. In [3, 4], based on the multiple linear copositive Lyapunov function method, the stability problem for switched positive linear systems with the average dwell time switching is studied. By using the technique of adding a power integrator, $\mathrm{Fu}$ et al. [5] discuss the finite-time control problem, and an adaptive controller is constructed to achieve the globally finite-time stabilization of the switched nonlinear systems with the powers of positive odd rational numbers. It is worth pointing out that the mentioned references focus on dealing with the control problem for the switched systems without unknown system functions.

It is noted that in many practical industrial processes, not all the system functions can be accurately modeled. Thus, an important problem is to overcome the foregoing uncertainties. In recent years, some approaches have been proposed to overcome the restriction and a large amount of encouraging results have been reported [11-24]. When the considered systems are unknown, neural networks (NNs)/fuzzy logic systems are often used to approximate the uncertain function to construct a controller. Most works on adaptive NN control are based on backstepping technique in [15-24]. For example, a NN-based adaptive controller has been designed with backstepping method, and NNbased design methods warrant the semiglobal stability for the nonlinear systems in a strict-feedback form in [15]. In [16], a backstepping controller containing a recurrent-neuralnetwork-based uncertainty observer and a robust controller has been developed to address the position control problem 
for induction servomotors. By utilizing backstepping technique and NN appropriate method in $[17,18]$, the stability problem of a class of stochastic nonlinear strict-feedback systems has been explored. It is worth noting that the aforementioned results on adaptive NN control design only consider the uncertain nonswitched nonlinear systems. Recently, several works have reported on uncertain switched nonlinear systems [25-27]. For uncertain nonlinear multiagent systems, distributed finite-time control scheme and cooperative adaptive neural partial tracking errors constrained control have been addressed in [28] and [29], respectively.

In addition, input delay plays an increasing important role in the real engineering systems. It has been attracted much attention, and numerous results have been presented in [3038]. For example, in [30], the augmented complete LyapunovKrasovskii functional method has been adopted to solve the stability issue for linear systems with input delay. Based on the stability conditions proposed in [35], a state transformation technique has been presented to tackle the stability problem for linear systems with input delays. In [31], an improved reciprocally convex approach has been proposed to achieve the asymptotic stabilization of the continuous-time systems with time-varying input delays. With respect to the switched systems with input delay, a few results have been reported in [37, 38].

Inspired by the above discussions, this paper addresses the adaptive neural tracking control problem for a class of uncertain switched systems subject to input delay. The main work of this paper can be summarized as follows. (i) By introducing some nonnegative switching functions, the desired adaptive neural controller is developed, and it is guaranteed that the tracking error can achieve the accuracy which can be adjusted based on the actual demands. (ii) The Pade approximation approach is borrowed to deal with the problem of input delay in the considered systems. (iii) In each backstepping design, $\mathrm{NN}$ is employed to approximate the simultaneous domination function rather than the switched system function. Thus, the number of adaptive learning parameters can be reduced.

\section{Preliminaries}

This section introduces some preliminaries on the system stability and NN approximation theory.

Definition 1 ((SGUUB) [39]). For a general nonlinear system

$$
\begin{aligned}
\dot{x} & =f(x, t), \\
x\left(t_{0}\right) & =x_{0},
\end{aligned}
$$

where $x(t) \in R^{n}$ is the system state, $f: R^{n} \times\left[t_{0}, \infty\right] \longrightarrow R^{n}$ is a continuous vector-valued function, and $t_{0}$ and $x_{0} \in R^{n}$ denote the initial time and the initial state vector, respectively. If there exists a compact set $U \in R^{n}$ such that for all $x_{0} \in U$, there exists a $\delta>0$ and a number $T\left(\delta, x_{0}\right)$ such that $\|x(t)\|<$ $\delta$ for all $t \geq t_{0}+T\left(\delta, x_{0}\right)$; we say that the solution of this system is semiglobally uniformly ultimately bounded (SGUUB).
Lemma 2 ((Young's inequality) [39]). For $\forall(x, y) \in R^{2}$, the following inequality holds:

$$
x y \leq \frac{\iota^{p}}{p}|x|^{p}+\frac{1}{q \iota^{q}}|y|^{q},
$$

where $\iota>0, p>1, q>1$, and $(p-1)(q-1)=1$.

The following switching functions are introduced and they will be used to design the desired adaptive neural controller.

$$
\mathcal{N}_{m}^{\epsilon}(e)= \begin{cases}\frac{(|e|-\epsilon)^{m}}{m !}, & |e|>\epsilon \\ 0, & |e| \leq \epsilon\end{cases}
$$

and

$$
\begin{aligned}
\mathcal{S}_{m}^{\epsilon}(e) & |e| \geq \epsilon \\
= & \begin{array}{ll}
\operatorname{sgn}(e), & |e|<\epsilon, \\
1-2 \cos ^{m}\left(\frac{\pi}{2} \sin ^{m}\left(\frac{\pi}{4 \epsilon}(e+\epsilon)\right)\right),
\end{array}
\end{aligned}
$$

where $\epsilon>0$ is the given accuracy and $m$ is a positive integer.

The main characteristics of functions $\mathcal{N}_{m}^{\epsilon}(e)$ and $\mathcal{S}_{m}^{\epsilon}(e)$ are given by the following lemma.

Lemma 3 (see [40]). Functions $\mathcal{N}_{m}^{\epsilon}(e)$ and $\mathcal{S}_{m}^{\epsilon}(e)$ have the following properties:

(1) $\mathcal{N}_{m}^{\epsilon}(e) \in \mathscr{C}^{m}$ and $\mathcal{S}_{m}^{\epsilon}(e) \in \mathscr{C}^{m}$, where $\mathscr{C}^{m}$ is the set of $m$-th order continuously differentiable functions.

(2) For $l=1,2, \ldots, m$, the $l$-th order derivatives of $\mathcal{N}_{m+1}^{\epsilon}(e)$ are

$$
\frac{d^{l} \mathcal{N}_{m+1}^{\epsilon}(e)}{d e^{l}}=\mathcal{N}_{m-l+1}^{\epsilon}(e)\left[\mathcal{S}_{m-l+1}^{\epsilon}(e)\right]^{l} .
$$

(3) $\mathscr{N}_{m+1}^{\epsilon}(e)$ is a nonnegative function, and when and only when $|e| \leq \epsilon, \mathcal{N}_{m+1}^{\epsilon}(e)=0$.

(4) For $l=1,2, \ldots, m, \mathcal{N}_{l}^{\epsilon}(e)\left[\mathcal{S}_{l}^{\epsilon}(e)\right]^{2}=\mathcal{N}_{l}^{\epsilon}(e)$.

In addition, in this paper, some unknown continuous functions $F_{i}\left(X_{i}\right), i=1,2, \ldots, m$, which will be defined later, are adopted to design the desired controller, and some RBF NNs are used to approximate these functions on a compact set $\Omega_{i}$, i.e.,

$$
F_{i}\left(X_{i}\right)=W_{i}^{T} S_{i}\left(X_{i}\right)+\varepsilon_{i}\left(X_{i}\right),
$$

where the input vectors $X_{i} \in \Omega_{i} \subset R^{m}$, weight vectors $W_{i} \in R^{l}$, the NN node number $l>1, \varepsilon_{i}\left(X_{i}\right)$ are the $N N$ inherent approximation errors which are bounded over the compact sets, i.e., $\left|\varepsilon_{i}\left(X_{i}\right)\right| \leq \varepsilon_{i}^{*}$ with unknown constants $\varepsilon_{i}^{*}$, and $S\left(X_{i}\right)=\left[s_{1}\left(X_{i}\right), \ldots, s_{l}\left(X_{i}\right)\right]^{T}: \Omega_{i} \longrightarrow R^{l}$ are known smooth vector functions with $s_{q}\left(X_{i}\right)$ being chosen as the commonly used Gaussian functions, which have the form

$$
s_{q}\left(X_{i}\right)=\exp \left[\frac{-\left(X_{i}-\mu_{q}\right)^{T}\left(X_{i}-\mu_{q}\right)}{\eta^{2}}\right] \text {, }
$$


where $\mu_{q}=\left[\mu_{q 1}, \ldots, \mu_{q m}\right]^{T}$ are the centers of the receptive field and $\eta_{q}>0$ are the spreads of the Gaussian functions. The optimal weight vector $W_{i}$ is defined as

$$
W_{i}=\arg \min _{\widehat{W}_{i} \in R^{l}}\left\{\sup _{X_{i} \in \Omega_{i}}\left|F\left(X_{i}\right)-\widehat{W}_{i}^{T} S_{i}\left(X_{i}\right)\right|\right\},
$$

where $\widehat{W}_{i}$ is the estimate of $W_{i}$.

\section{Problem Formulation, Control Design, and Stability Analysis}

3.1. Problem Description. Consider a class of switched nonlinear systems described by

$$
\begin{aligned}
\dot{\chi}_{i} & =\chi_{i+1}+f_{i}^{\sigma(t)}\left(\bar{\chi}_{i}\right), \quad i=1, \ldots, m-1 \\
\dot{\chi}_{m} & =u(t-\tau)+f_{m}^{\sigma(t)}\left(\bar{\chi}_{m}\right) \\
y & =\chi_{1}
\end{aligned}
$$

where $\chi=\left[\chi_{1}, \chi_{2}, \ldots, \chi_{m}\right]^{\mathrm{T}} \in R^{m}$ is the state vector. $y \in R$ and $u \in R$ denote the system output and the control input, respectively. $\sigma(t):[0,+\infty) \longrightarrow \mathscr{M}=\{1,2, \ldots, n\}$ represents a switching signal, and $f_{i}^{k}: R^{i} \longrightarrow R$ are unknown continuous functions for $i=1,2, \ldots, m, k=1,2, \ldots, n$. $\tau$ represents the delayed time.

To deal with the problem of input delay in system (55), the Pade approximation approach used in [35] is introduced. Subsequently, we can have

$$
\begin{aligned}
\mathscr{L}[u(t-\tau)] & =\exp (-\tau v) \mathscr{L}[u(t)] \\
& =\frac{\exp (-\tau v / 2)}{\exp (\tau v / 2)} \mathscr{L}[u(t)] \\
& \approx \frac{1-\tau v / 2}{1+\tau v / 2} \mathscr{L}[u(t)],
\end{aligned}
$$

where $\mathscr{L}[u(t)]$ is the Laplace transform of $u(t)$ and $v$ is Laplace variable. For further analysis, a new variable $\chi_{m+1}$ is proposed and it conforms to the following relation

$$
\mathscr{L}[u(t)] \frac{1-\tau v / 2}{1+\tau v / 2}=\mathscr{L}\left[\chi_{m+1}(t)\right]-\mathscr{L}[u(t)] .
$$

Then the following equation is obtained

$$
u-\frac{\tau}{2} \dot{u}=\chi_{m+1}+\frac{\tau}{2} \dot{\chi}_{m+1}-u-\frac{\tau}{2} \dot{u} .
$$

That is, we have the following equation

$$
\dot{\chi}_{m+1}=-\kappa \chi_{m+1}+2 \kappa u
$$

where $\kappa=2 / \tau$.
Remark 4. In this paper, Pade approximation method is introduced to deal with small delay. Since Pade approximation has some limitations in handling delay, the proposed scheme cannot work in large-delay case. Relaxed control design for systems with long delay and actuators saturation deserves further investigation.

Based on the above transformation, system (6) can be rewritten as follows

$$
\begin{aligned}
\dot{\chi}_{i} & =\chi_{i+1}+f_{i}^{\sigma(t)}\left(\bar{\chi}_{i}\right), \quad i=1, \ldots, m-1 \\
\dot{\chi}_{m} & =\chi_{m+1}-u+f_{m}^{\sigma(t)}\left(\bar{\chi}_{m}\right) \\
\dot{\chi}_{m+1} & =-\kappa \chi_{m+1}+2 \kappa u \\
y & =\chi_{1} .
\end{aligned}
$$

The control objective of this paper is addressed as follows: for a given reference signal $y_{r}(t)$, to develop an adaptive neural controller $u$ such that

(i) all the closed-loop signals remain SGUUB;

(ii) the tracking error $\left|e_{1}\right|=\left|y-y_{r}\right| \leq \epsilon$ as $t \longrightarrow \infty$.

Remark 5. In fact, some control schemes have been proposed for uncertain systems to achieve the objective of ensuring that the tracking error converges to the accuracy given a priori according to the requirement; e.g., see $[6,40]$. However, this paper studies the control problem for a class of uncertain switched systems with input delay. For such more general system, the existing control methods cannot be directly employed to solve this control issue. As far as the authors know, this is the first work to address the practical control for uncertain switched systems with input delay.

To design the desired adaptive neural controller, the following assumption is given.

Assumption 6. The reference signal $y_{r}(t)$ and its derivative $y_{r}^{(i)}(t)$ are continuous and bounded for $i=1,2, \ldots, m$.

3.2. Adaptive Neural Controller Design and Stability Analysis. To develop the desired control scheme for system (14), the following error variables are defined

$$
\begin{aligned}
e_{1} & =\chi_{1}-y_{r}, \\
e_{i} & =\chi_{i}-\alpha_{i-1}, \quad i=2, \ldots, m-1 \\
e_{m} & =\chi_{m}-\alpha_{m-1}+\frac{1}{\kappa} \chi_{m+1},
\end{aligned}
$$

where $\alpha_{i}, i=1, \ldots, m-1$, denote the virtual control variables and will be designed later.

Following the adaptive backstepping control method, we present the design process of the desired controller and the adaptation laws. 
Step 1. Consider the error variable $e_{1}$ in (15) and the first subsystem in (14). Then, we have

$$
\dot{e}_{1}=\dot{\chi}_{1}-\dot{y}_{r}=\chi_{2}+f_{1}^{\sigma(t)}\left(\bar{\chi}_{1}\right)-\dot{y}_{r}
$$

Select the following nonnegative function

$$
V_{1}=m ! \mathcal{N}_{m+1}^{\epsilon}\left(e_{1}\right)
$$

and then along the trajectory of (16), by using Lemma 3, the time derivative of $V_{1}$ is given as

$$
\begin{aligned}
& \frac{d V_{1}}{d t}=\frac{d \mathcal{N}_{m+1}^{\epsilon}\left(e_{1}\right)}{d e_{1}} \dot{e}_{1} m !=\mathcal{N}_{m}^{\epsilon}\left(e_{1}\right) \mathcal{S}_{m}^{\epsilon}\left(e_{1}\right) \\
& \cdot m !\left[\chi_{2}+f_{1}^{\sigma(t)}\left(\bar{\chi}_{1}\right)-\dot{y}_{r}\right] \leq \mathcal{N}_{m}^{\epsilon}\left(e_{1}\right) \mathcal{S}_{m}^{\epsilon}\left(e_{1}\right) \\
& \cdot m !\left[e_{2}+\alpha_{1}+\mathcal{S}_{m}^{\epsilon}\left(e_{1}\right) G_{1}\left(\bar{\chi}_{1}\right)-\dot{y}_{r}\right],
\end{aligned}
$$

where $G_{1}\left(\bar{\chi}_{1}\right)$ is a simultaneous domination function which is continuous and unknown. For example, here it can be selected as $G_{1}\left(\bar{\chi}_{1}\right)=\sqrt{\sum_{1}^{n}\left[f_{1}^{k}\left(\bar{\chi}_{1}\right)\right]^{2}+1}$. Since $f_{1}^{k}\left(\bar{\chi}_{1}\right)$ is unknown, it is obvious that $G_{1}\left(\bar{\chi}_{1}\right)$ is unknown and it cannot be used to design the controller directly. As shown in (6), an $\mathrm{RBF} N \mathrm{NN}$ is employed to approximate $F_{1}\left(X_{1}\right)$ online, and then one can have

$$
\begin{aligned}
\frac{d V_{1}}{d t} \leq \mathcal{N}_{m}^{\epsilon}\left(e_{1}\right) \mathcal{S}_{m}^{\epsilon}\left(e_{1}\right) \\
\cdot m !\left[e_{2}+\alpha_{1}+\mathcal{S}_{m}^{\epsilon}\left(e_{1}\right) G_{1}\left(\bar{\chi}_{1}\right)-\dot{y}_{r}\right]=\mathcal{N}_{m}^{\epsilon}\left(e_{1}\right) \\
\cdot \mathcal{S}_{m}^{\epsilon}\left(e_{1}\right) m !\left[e_{2}+\alpha_{1}+F_{1}\left(X_{1}\right)\right]=\mathcal{N}_{m}^{\epsilon}\left(e_{1}\right) \\
\cdot \mathcal{S}_{m}^{\epsilon}\left(e_{1}\right) m !\left[e_{2}+\alpha_{1}+W_{1}^{T} S_{1}\left(X_{1}\right)+\varepsilon_{1}\left(X_{1}\right)\right]
\end{aligned}
$$

where we define $F_{1}\left(X_{1}\right)=\mathcal{S}_{m}^{\epsilon}\left(e_{1}\right) G_{1}\left(\bar{\chi}_{1}\right)-\dot{y}_{r}$ with $X_{1}=$ $\left[\chi_{1}, y_{r}, \dot{y}_{r}\right]^{T} \in \Omega_{1} \subseteq R^{3}$.

The Young's inequality is used for the following analysis:

$$
\begin{gathered}
\mathcal{N}_{m}^{\epsilon}\left(e_{1}\right) \mathcal{S}_{m}^{\epsilon}\left(e_{1}\right) m ! W_{1}^{T} S_{1}\left(X_{1}\right) \leq \mathcal{N}_{m}^{\epsilon}\left(e_{1}\right)\left[\mathcal{S}_{m}^{\epsilon}\left(e_{1}\right)\right]^{2} \\
\cdot m !\left\|W_{1}\right\|\left\|S_{1}\left(X_{1}\right)\right\| \leq \mathcal{N}_{m}^{\epsilon}\left(e_{1}\right)\left[\mathcal{S}_{m}^{\epsilon}\left(e_{1}\right)\right]^{2} \\
\cdot m !\left(\frac{1}{2 a_{1}^{2}} \psi_{1}\left\|S_{1}\left(X_{1}\right)\right\|^{2}+\frac{1}{2} a_{1}^{2}\right)
\end{gathered}
$$

where $\psi_{1}:=\left\|W_{1}\right\|^{2}$ and $a_{1}>0$ is a design parameter, and

$$
\begin{aligned}
& \mathcal{N}_{m}^{\epsilon}\left(e_{1}\right) \mathcal{S}_{m}^{\epsilon}\left(e_{1}\right) m ! \varepsilon_{1}\left(X_{1}\right) \\
& \quad \leq \mathcal{N}_{m}^{\epsilon}\left(e_{1}\right)\left[\mathcal{S}_{m}^{\epsilon}\left(e_{1}\right)\right]^{2} m ! \varepsilon_{1}^{*} .
\end{aligned}
$$

Substituting (20) and (21) into (19) yields

$$
\begin{aligned}
& \frac{d V_{1}}{d t} \leq \mathcal{N}_{m}^{\epsilon}\left(e_{1}\right) \mathcal{S}_{m}^{\epsilon}\left(e_{1}\right) m !\left[e_{2}+\alpha_{1}\right. \\
& +\frac{1}{2 a_{1}^{2}} \psi_{1}\left\|S_{1}\left(X_{1}\right)\right\|^{2} \mathcal{S}_{m}^{\epsilon}\left(e_{1}\right)+\frac{1}{2} a_{1}^{2} \mathcal{S}_{m}^{\epsilon}\left(e_{1}\right) \\
& \left.+\mathcal{S}_{m}^{\epsilon}\left(e_{1}\right) \varepsilon_{1}^{*}\right]=\mathcal{N}_{m}^{\epsilon}\left(e_{1}\right) \mathcal{S}_{m}^{\epsilon}\left(e_{1}\right) m !\left[e_{2}+\alpha_{1}\right. \\
& \left.+\frac{1}{2 a_{1}^{2}} \psi_{1}\left\|S_{1}\left(X_{1}\right)\right\|^{2} \delta_{m}^{\epsilon}\left(e_{1}\right)+\delta_{m}^{\epsilon}\left(e_{1}\right) \rho_{1}\right]
\end{aligned}
$$

where $\rho_{1}:=(1 / 2) a_{1}^{2}+\varepsilon_{1}^{*}$ is an unknown constant.

The first virtual controller is designed from the above information.

$$
\begin{aligned}
\alpha_{1}= & -\left(k_{1}+\frac{1}{4}\right) \mathcal{N}_{m}^{\epsilon}\left(e_{1}\right) \mathcal{S}_{m}^{\epsilon}\left(e_{1}\right) m ! \\
& -\frac{1}{2 a_{1}^{2}} \widehat{\psi_{1}}\left\|S_{1}\left(X_{1}\right)\right\|^{2} \mathcal{S}_{m}^{\epsilon}\left(e_{1}\right)-\widehat{\rho_{1}} \mathcal{S}_{m}^{\epsilon}\left(e_{1}\right) \\
& -(\epsilon+1) \mathcal{S}_{m}^{\epsilon}\left(e_{1}\right),
\end{aligned}
$$

where $k_{1}$ is a positive constant and $\widehat{\psi_{1}}$ and $\widehat{\rho_{1}}$ denote the estimates of $\psi_{1}$ and $\rho_{1}$.

Obviously, (22) can be changed into

$$
\begin{aligned}
& \frac{d V_{1}}{d t} \leq \mathcal{N}_{m}^{\epsilon}\left(e_{1}\right) \mathcal{S}_{m}^{\epsilon}\left(e_{1}\right) m !\left[e_{2}\right. \\
& -\left(k_{1}+\frac{1}{4}\right) \mathcal{N}_{m}^{\epsilon}\left(e_{1}\right) \mathcal{S}_{m}^{\epsilon}\left(e_{1}\right) m ! \\
& -\frac{1}{2 a_{1}^{2}} \widehat{\psi_{1}}\left\|S_{1}\left(X_{1}\right)\right\|^{2} \mathcal{S}_{m}^{\epsilon}\left(e_{1}\right)-\widehat{\rho}_{1} \mathcal{S}_{m}^{\epsilon}\left(e_{1}\right) \\
& -(\epsilon+1) \mathcal{S}_{m}^{\epsilon}\left(e_{1}\right)+\frac{1}{2 a_{1}^{2}} \psi_{1}\left\|S_{1}\left(X_{1}\right)\right\|^{2} \mathcal{S}_{m}^{\epsilon}\left(e_{1}\right) \\
& \left.+\rho_{1} \mathcal{S}_{m}^{\epsilon}\left(e_{1}\right)\right] \leq-\left(k_{1}+\frac{1}{4}\right)\left[\mathcal{N}_{m}^{\epsilon}\left(e_{1}\right) m !\right]^{2}+\frac{1}{2 a_{1}^{2}} \\
& \cdot \widetilde{\psi}_{1} \mathcal{N}_{m}^{\epsilon}\left(e_{1}\right)\left[\mathcal{S}_{m}^{\epsilon}\left(e_{1}\right)\right]^{2} m !\left\|S_{1}\left(X_{1}\right)\right\|^{2}+\tilde{\rho}_{1} \mathcal{N}_{m}^{\epsilon}\left(e_{1}\right) \\
& \cdot\left[\mathcal{S}_{m}^{\epsilon}\left(e_{1}\right)\right]^{2} m !+\mathcal{N}_{m}^{\epsilon}\left(e_{1}\right) m !\left[\left|e_{2}\right|-(\epsilon+1)\right]
\end{aligned}
$$

with estimate errors $\widetilde{\psi}_{1}:=\psi_{1}-\widehat{\psi}_{1}$ and $\widetilde{\rho}_{1}:=\rho_{1}-\widehat{\rho}_{1}$.

For further study, we establish the nonnegative function as below

$$
\bar{V}_{1}=V_{1}+\frac{1}{2 \lambda_{1}} \widetilde{\psi}_{1}^{2}+\frac{1}{2 \gamma_{1}} \widetilde{\rho}_{1}^{2},
$$

where $\lambda_{1}>0$ and $\gamma_{1}>0$ are design parameters. We choose the adaptation laws as follows: 


$$
\begin{aligned}
& \dot{\hat{\psi}}_{1}=\frac{\lambda_{1}}{2 a_{1}^{2}} \mathcal{N}_{m}^{\epsilon}\left(e_{1}\right)\left\|S_{1}\left(X_{1}\right)\right\|^{2}\left[\mathcal{S}_{m}^{\epsilon}\left(e_{1}\right)\right]^{2} m ! \\
& \dot{\hat{\rho}}_{1}=\gamma_{1} \mathcal{N}_{m}^{\epsilon}\left(e_{1}\right)\left[\mathcal{S}_{m}^{\epsilon}\left(e_{1}\right)\right]^{2} m ! .
\end{aligned}
$$

Then we can obtain

$$
\begin{aligned}
& \frac{d \bar{V}_{1}}{d t}=\frac{d V_{1}}{d t}-\frac{1}{\lambda_{1}} \widetilde{\psi}_{1} \dot{\hat{\psi}}_{1}-\frac{1}{\gamma_{1}} \widetilde{\rho}_{1} \dot{\hat{\rho}}_{1} \leq-\left(k_{1}+\frac{1}{4}\right) \\
& \cdot\left[\mathcal{N}_{m}^{\epsilon}\left(e_{1}\right) m !\right]^{2}-\frac{1}{\lambda_{1}} \widetilde{\psi}_{1}\left\{\dot{\hat{\psi}}_{1}\right. \\
& \left.-\frac{\lambda_{1}}{2 a_{1}^{2}}\left\|S_{1}\left(X_{1}\right)\right\|^{2} \mathcal{N}_{m}^{\epsilon}\left(e_{1}\right)\left[\mathcal{S}_{m}^{\epsilon}\left(e_{1}\right)\right]^{2} m !\right\}-\frac{1}{\gamma_{1}} \\
& \cdot \widetilde{\rho}_{1}\left\{\dot{\hat{\rho}}_{1}-\gamma_{1} \mathcal{N}_{m}^{\epsilon}\left(e_{1}\right)\left[\mathcal{S}_{m}^{\epsilon}\left(e_{1}\right)\right]^{2} m !\right\}+\mathcal{N}_{m}^{\epsilon}\left(e_{1}\right) \\
& \cdot m !\left[\left|e_{2}\right|-(\epsilon+1)\right]=-\left(k_{1}+\frac{1}{4}\right)\left[\mathcal{N}_{m}^{\epsilon}\left(e_{1}\right) m !\right]^{2} \\
& +\mathcal{N}_{m}^{\epsilon}\left(e_{1}\right) m !\left[\left|e_{2}\right|-(\epsilon+1)\right] .
\end{aligned}
$$

$$
\begin{aligned}
\frac{d V_{i}}{d t} & =\frac{d \bar{V}_{i-1}}{d t}+\mathcal{N}_{m-i+1}^{\epsilon}\left(e_{i}\right) \mathcal{S}_{m-i+1}^{\epsilon}\left(e_{i}\right)(m-i+1) ! \dot{e}_{i} \\
& =\frac{d \bar{V}_{i-1}}{d t}+\mathcal{N}_{m-i+1}^{\epsilon}\left(e_{i}\right) \delta_{m-i+1}^{\epsilon}\left(e_{i}\right)(m-i+1) !\left\{e_{i+1}+\alpha_{i}+f_{i}^{\sigma(t)}\left(\bar{\chi}_{i}\right)-\Lambda_{i-1}-\sum_{j=1}^{i-1} \frac{\partial \alpha_{i-1}}{\partial \chi_{j}}\left[\chi_{j+1}+f_{j}^{\sigma(t)}\left(\bar{\chi}_{j}\right)\right]\right\} .
\end{aligned}
$$

Meanwhile the following inequality holds

$$
\begin{aligned}
& -\mathcal{N}_{m-i+1}^{\epsilon}\left(e_{i}\right) \mathcal{S}_{m-i+1}^{\epsilon}\left(e_{i}\right) \\
& \cdot(m-i+1) ! \sum_{j=1}^{i-1} \frac{\partial \alpha_{i-1}}{\partial \chi_{j}} f_{j}^{\sigma(t)}\left(\bar{\chi}_{j}\right) \leq \mathcal{N}_{m-i+1}^{\epsilon}\left(e_{i}\right)
\end{aligned}
$$

Step $i(i=2,3, \ldots, m-1)$. The method of recursion is used in each step when the value of $i$ increases from 2 to $m-1$. Similar to Step 1, the time derivative of $e_{i}$ is

$$
\dot{e}_{i}=\dot{\chi}_{i}-\dot{\alpha}_{i-1}=\chi_{i+1}+f_{i}^{\sigma(t)}\left(\bar{\chi}_{i}\right)-\dot{\alpha}_{i-1} \text {, }
$$

where $\dot{\alpha}_{i-1}=\sum_{j=1}^{i-1}\left(\partial \alpha_{i-1} / \partial \chi_{j}\right)\left[\chi_{j+1}+f_{j}^{\sigma(t)}\left(\bar{\chi}_{j}\right)\right]+\Lambda_{i-1}$ with $\Lambda_{i-1}=\sum_{j=0}^{i-1}\left(\partial \alpha_{i-1} / \partial y_{d}^{(j)}\right) y_{d}^{(j+1)}+\sum_{j=1}^{i-1}\left(\partial \alpha_{i-1} / \partial \widehat{\psi}_{j}\right) \dot{\hat{\psi}}_{j}+$ $\sum_{j=1}^{i-1}\left(\partial \alpha_{i-1} / \partial \hat{\rho}_{j}\right) \dot{\hat{\rho}}_{j}$

Consider the following nonnegative functions

$$
\begin{aligned}
V_{i} & =\bar{V}_{i-1}+\mathcal{N}_{m-i+2}^{\epsilon}\left(e_{i}\right)(m-i+1) !, \\
\bar{V}_{i} & =V_{i}+\frac{1}{2 \lambda_{i}} \widetilde{\psi}_{i}^{2}+\frac{1}{2 \gamma_{i}} \widetilde{\rho}_{i}^{2},
\end{aligned}
$$

where $\lambda_{i}>0$ and $\gamma_{i}>0$ are design parameters, estimate errors $\widetilde{\psi}_{i}:=\psi_{i}-\widehat{\psi}_{i}$ and $\tilde{\rho}_{i}:=\rho_{i}-\widehat{\rho}_{i}$, and $\widehat{\psi_{i}}$ and $\widehat{\rho_{i}}$ denote the estimates of $\psi_{i}$ and $\rho_{i}$ which will be defined later.

Then we can obtain

$$
\begin{aligned}
\frac{d V_{i}}{d t} \leq & \frac{d \bar{V}_{i-1}}{d t} \\
& +\mathcal{N}_{m-i+1}^{\epsilon}\left(e_{i}\right) \delta_{m-i+1}^{\epsilon}\left(e_{i}\right)(m-i+1) !\left\{e_{i+1}+\alpha_{i}+\delta_{m-i+1}^{\epsilon}\left(e_{i}\right)\left[G_{i}\left(\bar{\chi}_{i}\right)+\sum_{j=1}^{i-1} \Gamma_{j}\left(\bar{\chi}_{j}\right)\right]-\Lambda_{i-1}-\sum_{j=1}^{i-1} \frac{\partial \alpha_{i-1}}{\partial \chi_{j}} \chi_{j+1}\right\}
\end{aligned}
$$

where $G_{i}\left(\bar{\chi}_{\dot{i}}\right)$ and $\Gamma_{j}\left(\overline{\chi_{j}}\right)$ are simultaneous domination functions which are continuous and unknown. For example, here they can be chosen as $G_{i}\left(\bar{\chi}_{i}\right)=\sqrt{\sum_{k=1}^{n}\left[f_{i}^{k}\left(\overline{\chi_{i}}\right)\right]^{2}+1}$, $\Gamma_{j}\left(\bar{\chi}_{j}\right)=\sqrt{\sum_{k=1}^{n}\left[\left(\partial \alpha_{i-1} / \partial \chi_{j}\right) f_{j}^{k}\left(\bar{\chi}_{j}\right)\right]^{2}+1}$. Define $F_{i}\left(X_{i}\right)=$ $\mathcal{S}_{m-i+1}^{\epsilon}\left(e_{i}\right)\left[G_{i}\left(\bar{\chi}_{i}\right)+\sum_{j=1}^{i-1} \Gamma_{j}\left(\overline{\chi_{j}}\right)\right]-\Lambda_{i-1}-\sum_{j=1}^{i-1}\left(\partial \alpha_{i-1} / \partial \chi_{j}\right) \chi_{j+1}$ with $X_{i}=\left[\bar{\chi}_{i}^{T}, \alpha_{i-1}, \partial \alpha_{i-1} / \partial \chi_{1}, \ldots, \partial \alpha_{i-1} / \partial \chi_{i-1}, \Lambda_{i-1}\right]^{T} \in \Omega_{i} \subseteq$
Substituting (31) into (30), we gain

$$
\begin{aligned}
& \cdot\left[\mathcal{S}_{m-i+1}^{\epsilon}\left(e_{i}\right)\right]^{2} \\
& \cdot(m-i+1) ! \sum_{j=1}^{i-1} \sqrt{\left[\frac{\partial \alpha_{i-1}}{\partial \chi_{j}} f_{j}^{\sigma(t)}\left(\bar{\chi}_{j}\right)\right]^{2}+1 .}
\end{aligned}
$$

$R^{2 i+1}$, and an RBF NN (6) is used to approximate $F_{i}\left(X_{i}\right)$ online. Based on this information, (32) can be changed as below

$$
\begin{aligned}
& \frac{d V_{i}}{d t} \leq \frac{d \bar{V}_{i-1}}{d t}+\mathcal{N}_{m-i+1}^{\epsilon}\left(e_{i}\right) \mathcal{S}_{m-i+1}^{\epsilon}\left(e_{i}\right) \\
& \cdot(m-i+1) !\left[e_{i+1}+\alpha_{i}+F_{i}\left(X_{i}\right)\right]=\frac{d \bar{V}_{i-1}}{d t}
\end{aligned}
$$




$$
\begin{aligned}
& +\mathcal{N}_{m-i+1}^{\epsilon}\left(e_{i}\right) \mathcal{S}_{m-i+1}^{\epsilon}\left(e_{i}\right) \\
& \cdot(m-i+1) !\left[e_{i+1}+\alpha_{i}+W_{i}^{T} S_{i}\left(X_{i}\right)+\varepsilon_{i}\left(X_{i}\right)\right] .
\end{aligned}
$$

Similar to (20) and (21), by using the Young's inequality, inequalities (34) and (35) can be gained easily:

$$
\begin{aligned}
& \mathcal{N}_{m-i+1}^{\epsilon}\left(e_{i}\right) \mathcal{S}_{m-i+1}^{\epsilon}\left(e_{i}\right)(m-i+1) ! W_{i}^{T} S_{i}\left(X_{i}\right) \\
& \quad \leq \mathcal{N}_{m-i+1}^{\epsilon}\left(e_{i}\right)\left[\mathcal{S}_{m-i+1}^{\epsilon}\left(e_{i}\right)\right]^{2} \\
& \cdot(m-i+1) !\left\|W_{i}^{T}\right\|\left\|S_{i}\left(X_{i}\right)\right\| \leq \mathcal{N}_{m-i+1}^{\epsilon}\left(e_{i}\right)
\end{aligned}
$$

$$
\begin{aligned}
& \cdot\left[\mathcal{S}_{m-i+1}^{\epsilon}\left(e_{i}\right)\right]^{2} \\
& \cdot(m-i+1) !\left(\frac{1}{2 a_{i}^{2}} \psi_{i}\left\|S_{i}\left(X_{i}\right)\right\|^{2}+\frac{1}{2} a_{i}^{2}\right)
\end{aligned}
$$

where $\psi_{i}=\left\|W_{i}\right\|^{2}, a_{i}>0$ is a design parameter, and

$$
\begin{aligned}
& \mathscr{N}_{m-i+1}^{\epsilon}\left(e_{i}\right) \mathcal{S}_{m-i+1}^{\epsilon}\left(e_{i}\right)(m-i+1) ! \varepsilon_{i}\left(X_{i}\right) \\
& \quad \leq \mathcal{N}_{m-i+1}^{\epsilon}\left(e_{i}\right)\left[\mathcal{S}_{m-i+1}^{\epsilon}\left(e_{i}\right)\right]^{2}(m-i+1) ! \varepsilon_{i}^{*}
\end{aligned}
$$

Substituting (34) and (35) into (33) yields

$$
\begin{aligned}
\frac{d V_{i}}{d t} \leq & \frac{d \bar{V}_{i-1}}{d t} \\
& +\mathcal{N}_{m-i+1}^{\epsilon}\left(e_{i}\right) \mathcal{S}_{m-i+1}^{\epsilon}\left(e_{i}\right)(m-i+1) !\left[e_{i+1}+\alpha_{i}+\left(\frac{1}{2 a_{i}^{2}} \psi_{i}\left\|S_{i}\left(X_{i}\right)\right\|^{2}+\frac{1}{2} a_{i}^{2}\right) \mathcal{S}_{m-i+1}^{\epsilon}\left(e_{i}\right)+\mathcal{S}_{m-i+1}^{\epsilon}\left(e_{i}\right) \varepsilon_{i}^{*}\right] \\
= & \frac{d \bar{V}_{i-1}}{d t}+\mathcal{N}_{m-i+1}^{\epsilon}\left(e_{i}\right) \mathcal{S}_{m-i+1}^{\epsilon}\left(e_{i}\right)(m-i+1) !\left[e_{i+1}+\alpha_{i}+\frac{1}{2 a_{i}^{2}} \psi_{i}\left\|S_{i}\left(X_{i}\right)\right\|^{2} \mathcal{S}_{m-i+1}^{\epsilon}\left(e_{i}\right)+\mathcal{S}_{m-i+1}^{\epsilon}\left(e_{i}\right) \rho_{i}\right],
\end{aligned}
$$

where $\rho_{i}=(1 / 2) a_{i}^{2}+\varepsilon_{i}^{*}$ is an unknown constant.

We construct the $i$-th virtual controller as follows:

$$
\begin{aligned}
\alpha_{i}= & -\left(k_{i}+\frac{5}{4}\right) \mathcal{N}_{m-i+1}^{\epsilon}\left(e_{i}\right) \mathcal{S}_{m-i+1}^{\epsilon}\left(e_{i}\right)(m-i+1) ! \\
& -\frac{1}{2 a_{i}^{2}} \widehat{\psi}_{i}\left\|S_{i}\left(X_{i}\right)\right\|^{2} \mathcal{S}_{m-i+1}^{\epsilon}\left(e_{i}\right)-\mathcal{S}_{m-i+1}^{\epsilon}\left(e_{i}\right) \widehat{\rho}_{i} \\
& -(\epsilon+1) \mathcal{S}_{m-i+1}^{\epsilon}\left(e_{i}\right) .
\end{aligned}
$$

Obviously, based on (37), the undermentioned inequality is true:

$$
\begin{aligned}
& \frac{d V_{i}}{d t} \leq \frac{d \bar{V}_{i-1}}{d t}+\mathscr{N}_{m-i+1}^{\epsilon}\left(e_{i}\right) \mathcal{S}_{m-i+1}^{\epsilon}\left(e_{i}\right)(m-i \\
& \quad+1) !\left[e_{i+1}+\frac{1}{2 a_{i}^{2}} \widetilde{\psi}_{i}\left\|S_{i}\left(X_{i}\right)\right\|^{2} \mathcal{S}_{m-i+1}^{\epsilon}\left(e_{i}\right)+\mathcal{S}_{m-i+1}^{\epsilon}\left(e_{i}\right) \widetilde{\rho}_{i}-(\epsilon+1) \mathcal{S}_{m-i+1}^{\epsilon}\left(e_{i}\right)-\left(k_{i}+\frac{5}{4}\right) \mathcal{N}_{m-i+1}^{\epsilon}\left(e_{i}\right) \mathcal{S}_{m-i+1}^{\epsilon}\left(e_{i}\right)(m-i+1) !\right] \\
& \quad \leq-\sum_{j=1}^{i} k_{j}\left[\mathcal{N}_{m-j+1}^{\epsilon}\left(e_{j}\right)(m-j+1) !\right]^{2}+\mathcal{N}_{m-i+1}^{\epsilon}\left(e_{i}\right)\left[\mathcal{S}_{m-i+1}^{\epsilon}\left(e_{i}\right)\right]^{2}(m-i+1) ! \times\left[\frac{1}{2 a_{i}^{2}} \widetilde{\psi}_{i}\left\|S_{i}\left(X_{i}\right)\right\|^{2}+\widetilde{\rho}_{i}\right]-\frac{1}{4}\left[\mathcal{N}_{m-i+2}^{\epsilon}\left(e_{i-1}\right)\right. \\
& \quad \cdot(m-i+2)]^{2}+\mathcal{N}_{m-i+2}^{\epsilon}\left(e_{i}\right)(m-i+2) !\left[\left|e_{i}\right|-(\epsilon+1)\right]-\frac{5}{4}\left[\mathcal{N}_{m-i+1}^{\epsilon}\left(e_{i}\right)(m-i+1)\right]^{2}+\mathcal{N}_{m-i+1}^{\epsilon}\left(e_{i}\right)(m-i \\
& \quad+1) !\left[e_{i+1}-(\epsilon+1)\right] .
\end{aligned}
$$

Let

$$
\begin{aligned}
\mathscr{U}_{i}= & -\frac{1}{4}\left[\mathcal{N}_{m-i+2}^{\epsilon}\left(e_{i-1}\right)(m-i+2)\right]^{2} \\
& +\mathcal{N}_{m-i+2}^{\epsilon}\left(e_{i-1}\right)(m-i+2) !\left[e_{i}-(\epsilon+1)\right] \\
& -\left[\mathcal{N}_{m-i+1}^{\epsilon}\left(e_{i}\right)(m-i+1)\right]^{2} .
\end{aligned}
$$

$$
\begin{aligned}
\mathcal{U}_{i} & \leq\left[\left|e_{i}\right|-(\epsilon+1)\right]^{2}-\left[\mathcal{N}_{m-i+1}^{\epsilon}\left(e_{i}\right)(m-i+1)\right]^{2} \\
& =\left[\left|e_{i}\right|-(\epsilon+1)\right]^{2}-\left[\left|e_{i}\right|-\epsilon\right]^{2(m-i+1)}
\end{aligned}
$$




$$
\begin{aligned}
& \leq\left(\left|e_{i}\right|-\epsilon\right)^{2}-\left[\left|e_{i}\right|-\epsilon\right]^{2(m-i+1)} \\
& =\left(\left|e_{i}\right|-\epsilon\right)^{2}\left[1-\left(\left|e_{i}\right|-\epsilon\right)\right]^{2(m-i+1)} \leq 0 .
\end{aligned}
$$

Hence, $\mathcal{U}_{i} \leq 0$ holds all the time.

Based on the above information, we choose

$$
\begin{aligned}
\dot{\widehat{\psi}}_{i} & =\frac{\lambda_{i}}{2 a_{i}^{2}} \mathcal{N}_{m-i+1}^{\epsilon}\left(e_{i}\right)\left[\mathcal{S}_{m-i+1}^{\epsilon}\left(e_{i}\right)\right]^{2} \\
& \cdot(m-i+1) !\left\|S_{i}\left(X_{i}\right)\right\|^{2} \\
\dot{\hat{\rho}}_{i} & =\gamma_{i} \mathcal{N}_{m-i+1}^{\epsilon}\left(e_{i}\right)\left[\mathcal{S}_{m-i+1}^{\epsilon}\left(e_{i}\right)\right]^{2}(m-i+1) !
\end{aligned}
$$

Thus, we can have

$$
\begin{aligned}
& \frac{d \bar{V}_{i}}{d t}=\frac{d V_{i}}{d t}-\frac{1}{\lambda_{i}} \widetilde{\psi}_{i} \dot{\hat{\psi}}_{i}-\frac{1}{\gamma_{i}} \widetilde{\rho}_{i} \dot{\hat{\rho}}_{i} \leq-\sum_{j=1}^{i} k_{j}\left[\mathcal{N}_{m-j+1}^{\epsilon}\left(e_{j}\right)\right. \\
& \cdot(m-j+1)]^{2}-\frac{1}{\lambda_{i}} \widetilde{\psi}\left\{\dot{\hat{\psi}}_{i}-\frac{\lambda_{i}}{2 a_{i}^{2}} \mathcal{N}_{m-i+1}^{\epsilon}\left(e_{i}\right)\right. \\
& \left.\cdot\left[\mathcal{S}_{m-i+1}^{\epsilon}\left(e_{i}\right)\right]^{2}(m-i+1) !\left\|S_{i}\left(X_{i}\right)\right\|^{2}\right\}-\frac{1}{\gamma_{i}} \widetilde{\rho}_{i}\left\{\dot{\hat{\rho}}_{i}\right. \\
& \left.-\gamma_{i} \mathcal{N}_{m-i+1}^{\epsilon}\left(e_{i}\right)\left[\mathcal{S}_{m-i+1}^{\epsilon}\left(e_{i}\right)\right]^{2}(m-i+1) !\right\} \\
& -\frac{1}{4}\left[\mathcal{N}_{m-i+1}^{\epsilon}\left(e_{i}\right)(m-i+1) !\right]^{2}+\mathcal{N}_{m-i+1}^{\epsilon}\left(e_{i}\right)(m \\
& -i+1) !\left[\left|e_{i+1}\right|-(\epsilon+1)\right] \\
& \leq-\sum_{j=1}^{i} k_{j}\left[\mathcal{N}_{m-j+1}^{\epsilon}\left(e_{j}\right)(m-j+1)\right]^{2} \\
& -\frac{1}{4}\left[\mathcal{N}_{m-i+1}^{\epsilon}\left(e_{i}\right)(m-i+1) !\right]^{2}+\mathcal{N}_{m-i+1}^{\epsilon}\left(e_{i}\right)(m \\
& -i+1) !\left[\left|e_{i+1}\right|-(\epsilon+1)\right] .
\end{aligned}
$$

Step $m$. Combining (14) and (15), we have

$$
\begin{aligned}
\dot{e}_{m} & =\dot{\chi}_{m}-\dot{\alpha}_{m-1}+\frac{1}{\kappa} \dot{\chi}_{m+1} \\
& =\chi_{m+1}-u+f_{m}^{\sigma(t)}\left(\bar{\chi}_{m}\right)-\dot{\alpha}_{m-1}+\frac{1}{\kappa} \dot{\chi}_{m+1},
\end{aligned}
$$

where $\dot{\alpha}_{m-1}=\sum_{j=1}^{m-1}\left(\partial \alpha_{m-1} / \partial \chi_{j}\right)\left[\chi_{j+1}+f_{j}^{\sigma(t)}\left(\bar{\chi}_{j}\right)\right]+\Lambda_{m-1}$ with $\Lambda_{m-1}=\sum_{j=0}^{m-1}\left(\partial \alpha_{m-1} / \partial y_{d}^{(j)}\right) y_{d}^{(j+1)}+\sum_{j=1}^{m-1}\left(\partial \alpha_{m-1} / \partial \widehat{\psi}_{j}\right) \dot{\hat{\psi}}_{j}+$ $\sum_{j=1}^{m-1}\left(\partial \alpha_{m-1} / \partial \hat{\rho}_{j}\right) \dot{\hat{\rho}}_{j}$.

Choose $\bar{V}_{m}=V_{m}+\left(1 / 2 \lambda_{m}\right) \tilde{\psi}_{m}^{2}+\left(1 / 2 \gamma_{m}\right) \tilde{\rho}_{m}^{2}, V_{m}=\bar{V}_{m-1}+$ $\mathcal{N}_{2}^{\epsilon}\left(e_{m}\right)$, where $\lambda_{m}>0$ and $\gamma_{m}>0$ are design parameters, and design the actual controller as below

$$
\begin{aligned}
u= & -\left(k_{m}+1\right) \mathcal{N}_{1}^{\epsilon}\left(e_{m}\right) \\
& -\frac{1}{2 a_{m}^{2}} \widehat{\psi}_{m}^{2}\left\|S_{m}\left(X_{m}\right)\right\|^{2} \mathcal{S}_{1}^{\epsilon}\left(e_{m}\right)-\mathcal{S}_{1}^{\epsilon}\left(e_{m}\right) \widehat{\rho}_{m} \\
& -(\epsilon+1) \mathcal{S}_{1}^{\epsilon}\left(e_{m}\right),
\end{aligned}
$$

where $a_{m}>0$ and $k_{m}>0$ are design parameters, estimate errors $\tilde{\psi}_{m}:=\psi_{m}-\widehat{\psi}_{m}$ and $\tilde{\rho}_{m}:=\rho_{m}-\widehat{\rho}_{m}$, and $\widehat{\psi}_{m}$ and $\hat{\rho}_{m}$ denote the estimates of $\psi_{m}$ and $\rho_{m}$ which will be defined later.

Similar to the design process of Step $i$, let $i=m$. Then we can have $\psi_{m}=\left\|W_{m}\right\|^{2}$ and $\rho_{m}=(1 / 2) a_{m}^{2}+\varepsilon_{m}^{*}$ where $\varepsilon_{m}^{*}$ is the upper bound of the RBF NN approximation error. The following adaptive laws are chosen

$$
\begin{aligned}
& \dot{\hat{\psi}}_{m}=\frac{\lambda_{m}}{2 a_{m}^{2}} \mathcal{N}_{1}^{\epsilon}\left(e_{m}\right)\left[\mathcal{S}_{1}^{\epsilon}\left(e_{m}\right)\right]^{2}\left\|S_{m}\left(X_{m}\right)\right\|^{2} \\
& \dot{\hat{\rho}}_{m}=\gamma_{m} \mathcal{N}_{1}^{\epsilon}\left(e_{m}\right)\left[\mathcal{S}_{1}^{\epsilon}\left(e_{m}\right)\right]^{2},
\end{aligned}
$$

where we define $X_{m}=\left[\bar{\chi}_{m}^{T}, \alpha_{m-1}\right.$, $\left.\partial \alpha_{m-1} / \partial \chi_{1}, \ldots, \partial \alpha_{m-1} / \partial \chi_{m-1}, \Lambda_{m-1}\right]^{T} \in \Omega_{m} \subseteq R^{2 m+1}$.

According to the above information, we have

$$
\begin{aligned}
\frac{d \bar{V}_{m}}{d t} \leq & -\sum_{j=1}^{m} k_{j}\left[\mathcal{N}_{m-j+1}^{\epsilon}\left(e_{j}\right)(m-j+1) !\right]^{2} \\
& -\frac{1}{4}\left[\mathcal{N}_{2}^{\epsilon}\left(e_{m-1}\right) 2 !\right]^{2}-\left[\mathcal{N}_{1}^{\epsilon}\left(e_{m}\right)\right]^{2} \\
& +\mathcal{N}_{2}^{\epsilon}\left(e_{m-1}\right) 2 !\left[\left|e_{m}\right|-(\epsilon+1)\right] \\
\leq & -\sum_{j=1}^{m} k_{j}\left[\mathcal{N}_{m-j+1}^{\epsilon}\left(e_{j}\right)(m-j+1) !\right]^{2} .
\end{aligned}
$$

Based on inequality (46), the main result of this paper is summarized by the following theorem.

Theorem 7. Under Assumption 6, consider the closed-loop system that comprises the plant (9), virtual control variables (23) and (37), and actual control law (44) with adaptive laws (26), (41), and (45). For the bounded initial condition on a compact set $\Omega_{0}$, assume there exist sufficiently large compact sets $\Omega_{i} \subseteq R^{2 i+1}, i=1,2, \ldots, m$, such that $X_{i} \in \Omega_{i}$ for all $t \geq 0$; the following statements hold.

(i) The tracking error $\left|e_{1}\right|=\left|y-y_{r}\right| \leq \epsilon$ as $t \longrightarrow \infty$.

(ii) All the closed-loop signals remain semiglobal bounded.

Proof. (i) From (46), by employing Barbalat's Lemma [41], we can easily conclude that the tracking error satisfies $\left|e_{1}\right|=\mid y-$ $y_{r} \mid \leq \epsilon$ as $t \longrightarrow \infty$.

(ii) Considering inequality (46), we can see that $\bar{V}_{m}$ is nonincreasing. Therefore, it can be concluded that, for all $1 \leq i \leq m$, error signals including $e_{i}(t), \widetilde{\rho}_{i}(t)$ and $\widetilde{\psi}_{i}(t)$ are bounded. To prove the boundedness of all the closed-loop signals, it can be shown that $\widehat{\rho}_{i}(t)=\rho_{i}-\widetilde{\rho}_{i}(t)$ and $\widehat{\psi}_{i}(t)=$ $\psi_{i}-\psi_{i}(t)$ are bounded according to the boundedness of $\tilde{\rho}_{i}(t)$ and $\widetilde{\psi}_{i}(t)$. Next, it follows from the boundedness of $e_{1}(t)$ and $y_{r}(t)$ that $\chi_{1}(t)=y_{r}(t)+e_{1}(t)$ is also bounded. Then, the boundedness of $\alpha_{1}$ given in (23) can be easily obtained. The boundedness of $e_{2}$ and $\alpha_{1}$ further implies that $\chi_{2}=e_{2}+\alpha_{1}$ is bounded. Continuing in the same fashion, we conclude that all the closed-loop signals are bounded. In addition, similar to the existing works on adaptive neural network control, the proposed control scheme just guarantees that the closed-loop 
system is semiglobally stable since the initial condition of the controlled system must remain on a compact set $\Omega_{0}$.

Remark 8. To show the semiglobal stability of the controlled system, the following analysis is presented. Firstly, from $\chi_{1}=$ $e_{1}+y_{r}$, we have $\left|\chi_{1}\right| \leq\left|e_{1}\right|+\left|y_{r}\right| \leq \epsilon+Y$, where $Y$ is the upper bound of reference signal $y_{r}$. Thus, the boundedness of $\chi_{1}$ is obtained as $t \longrightarrow \infty$. Furthermore, from the above analysis, it can be seen that $\widehat{\psi}_{1}$ and $\widehat{\rho}_{1}$ are bounded. Assume that $\left|\widehat{\psi}_{1}\right| \leq$ $\Psi_{1}$ and $\left|\hat{\rho}_{1}\right| \leq \Phi_{1}$ with positive constants $\Psi_{1}$ and $\Phi_{1}$. Since $\chi_{2}=e_{2}+\alpha_{1}$, then we can obtain the following inequality for $t \rightarrow \infty$,

$$
\begin{aligned}
\left|\chi_{2}\right| \leq\left|e_{2}\right|+\left|\alpha_{1}\right| & \\
\leq & 2 \epsilon+\left(k_{1}+\frac{1}{4}\right) \mathcal{N}_{m}^{\epsilon}\left(e_{1}\right) m !+\frac{1}{2 a_{1}^{2}} \Psi_{1} \bar{S}_{1}+\Phi_{1} \\
& +1=2 \epsilon+\frac{1}{2 a_{1}^{2}} \Psi_{1} \bar{S}_{1}+\Phi_{1}+1
\end{aligned}
$$

where $-1 \leq \mathcal{S}_{m}^{\epsilon}\left(e_{1}\right) \leq 1, \mathcal{N}_{m}^{\epsilon}\left(e_{1}\right)=0$ when $\left|e_{1}\right| \leq \epsilon$, and basis function $S_{1}\left(X_{1}\right)$ satisfies $\left\|S_{1}\left(X_{1}\right)\right\|^{2} \leq \bar{S}_{1}$ with a positive constant $\bar{S}_{1}$. From the above inequality, it can be seen that we may increase the size of the attraction region with increasing the gain $1 / a_{1}^{2}$.

By the same way, we can show that the signals $\chi_{3}, \ldots, \chi_{m}$ are semiglobally bounded.

Remark 9. For system (9) without input delay, some adaptive $\mathrm{NN} /$ fuzzy control schemes have been reported; e.g., see [25$27,42-44]$. For the control problem considered in this paper, two differences are summarized as follows. First, for the controlled switching system, the input delay phenomenon is considered. In addition, all the existing control methods for the uncertain switched system just can guarantee the tracking error converging to a small compact set, and the accurate size cannot be determined. This paper addresses the adaptive tracking control issue with the known tracking accuracy.

\section{Simulation Examples}

Example 1. Consider the following uncertain switched system

$$
\begin{aligned}
& \dot{\chi}_{1}=\chi_{2}+f_{1}^{\sigma(t)}\left(\bar{\chi}_{1}\right), \\
& \dot{\chi}_{2}=u(t-\tau)+f_{2}^{\sigma(t)}\left(\bar{\chi}_{2}\right) \\
& y=\chi_{1},
\end{aligned}
$$

where $\sigma(t)=1,2, f_{1}^{1}\left(\chi_{1}\right)=\sin \left(\chi_{1}\right) \cos \left(\chi_{1}\right), f_{2}^{1}\left(\chi_{1}\right)=\chi_{1}^{2}$, $f_{1}^{2}\left(\chi_{1}, \chi_{2}\right)=\sin \left(\chi_{1}\right) \cos \left(\chi_{2}\right)+\chi_{2}^{2}, f_{2}^{2}\left(\chi_{1}, \chi_{2}\right)=\chi_{1} \chi_{2}+\sin \left(\chi_{1}\right)$. The reference signal is given as $y_{r}(t)=2+\sin (2 t)+0.5 \cos (t)$. The tracking accuracy is assigned as $\epsilon=0.05$, i.e., $\mid y(t)-$ $y_{r}(t) \mid \leq 0.05$ as $t \longrightarrow \infty$.
According to the design process of Section 3, the virtual controller and the actual controller are presented as

$$
\begin{aligned}
\alpha_{1}= & -\left(k_{1}+\frac{1}{4}\right) \mathcal{N}_{2}^{\epsilon}\left(e_{1}\right) \mathcal{S}_{2}^{\epsilon}\left(e_{1}\right) 2 ! \\
& -\frac{1}{2 a_{1}^{2}} \widehat{\psi_{1}}\left\|S_{1}\left(X_{1}\right)\right\|^{2} \mathcal{S}_{2}^{\epsilon}\left(e_{1}\right)-\widehat{\rho_{1}} \mathcal{S}_{2}^{\epsilon}\left(e_{1}\right) \\
& -(\epsilon+1) \mathcal{S}_{2}^{\epsilon}\left(e_{1}\right),
\end{aligned}
$$

and

$$
\begin{aligned}
u= & -\left(k_{2}+1\right) \mathcal{N}_{1}^{\epsilon}\left(e_{2}\right)-\frac{1}{2 a_{2}^{2}} \widehat{\psi}_{2}^{2}\left\|S_{i}\left(X_{i}\right)\right\|^{2} \mathcal{S}_{1}^{\epsilon}\left(e_{2}\right) \\
& -\mathcal{S}_{1}^{\epsilon}\left(e_{2}\right) \hat{\rho}_{2}-(\epsilon+1) \mathcal{S}_{1}^{\epsilon}\left(e_{2}\right) .
\end{aligned}
$$

The adaptive laws are designed as

$$
\begin{aligned}
& \dot{\hat{\psi}}_{i}=\frac{\lambda_{i}}{2 a_{i}^{2}} \mathcal{N}_{3-i}^{\epsilon}\left(e_{i}\right)\left[\mathcal{S}_{3-i}^{\epsilon}\left(e_{i}\right)\right]^{2}(3-i) !\left\|S_{i}\left(X_{i}\right)\right\|^{2} \\
& \dot{\hat{\rho}}_{i}=\gamma_{i} \mathcal{N}_{3-i}^{\epsilon}\left(e_{i}\right)\left[\mathcal{S}_{3-i}^{\epsilon}\left(e_{i}\right)\right]^{2}(3-i) !, \quad i=1,2 .
\end{aligned}
$$

The design parameters of the adaptive neural controllers and learning laws are chosen as $k_{1}=k_{2}=3, a_{1}=a_{2}=$ $1, \gamma_{1}=7, \gamma_{2}=10$, and $\lambda_{1}=\lambda_{2}=15$. The input delay is $\tau=0.0012$. In the simulation, two RBF NNs are employed to approximate uncertain functions in the control scheme. The first RBF vector $S_{1}\left(X_{1}\right)$ contains 125 nodes with the centers $\mu_{q}(q=1,2, \ldots, 125)$ evenly placed on $[-1.7,1.7] \times[-3,3] \times$ $[-3,3]$ and the width $\eta=0.62$. The second RBF vector $S_{2}\left(X_{2}\right)$ contains 1875 nodes with the centers $\mu_{q}(q=1,2, \ldots, 1875)$ evenly placed on $[-1.7,1.7] \times[-3,3] \times[-3,3] \times[-7,7] \times[-7,7]$ and the width $\eta=0.38$.

The initial conditions are given as $\chi_{1}(0)=0.1, \chi_{2}(0)=$ 0.5 , and $\widehat{\rho}_{i}=\widehat{\psi}_{i}=0, i=1,2$. The simulation results are shown in Figures 1-4, and Figure 2 shows that the tracking error achieves the given accuracy after 20 seconds, which implies that the control objective is achieved by using the proposed control scheme.

To further show the superiority of the proposed control method, a comparison simulation is presented. Considering the control scheme developed in [42], we have the following controllers and parameters learning laws

$$
\begin{gathered}
u=\left(\frac{\hat{\theta}_{2}}{2 \zeta_{2, \text { min }}^{2}}+\lambda_{2}\right) z_{2}, \\
\alpha_{1}=\left(\frac{\hat{\theta}_{1}}{2 \zeta_{1, \text { min }}^{2}}+\lambda_{1}\right) z_{1},
\end{gathered}
$$

and

$$
\dot{\hat{\theta}}_{i}=\frac{r_{i}}{2 \zeta_{i, \text { min }}^{2}}-\beta_{i} \widehat{\theta}_{i}, \quad i=1,2
$$

where the definitions and values of $z_{i}, \lambda_{i}, r_{i}, \beta_{i}, \zeta_{i \text {,min }}$, and $\hat{\theta}_{i}$ can be found in [42]. For the simulation, the parameters are chosen as $r_{i}=\zeta_{i, \min }=1$ and $\beta_{i}=0$. The rest of the 


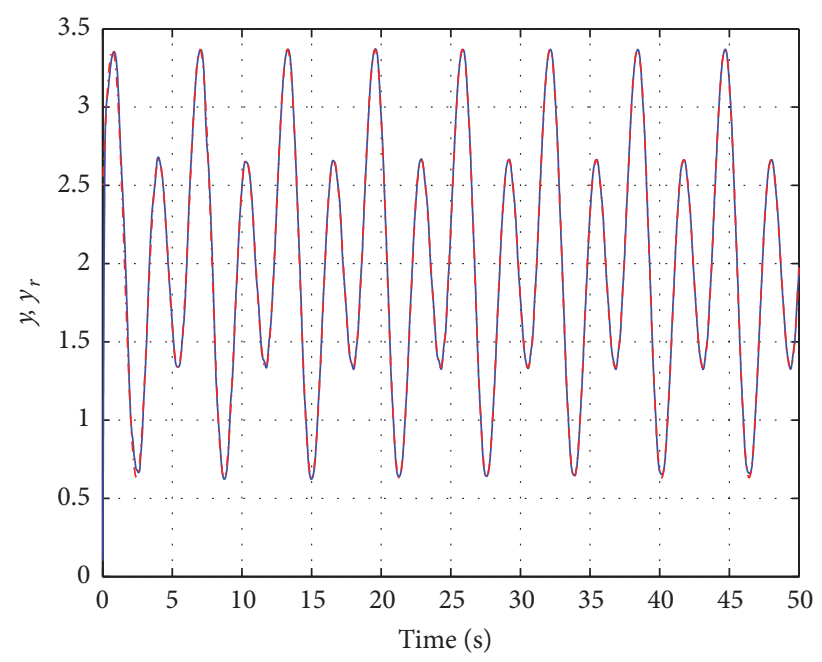

Figure 1: Trajectories of system output signal $y(t)$ (solid line) and reference signal $y_{r}(t)$ (dash-dotted line).

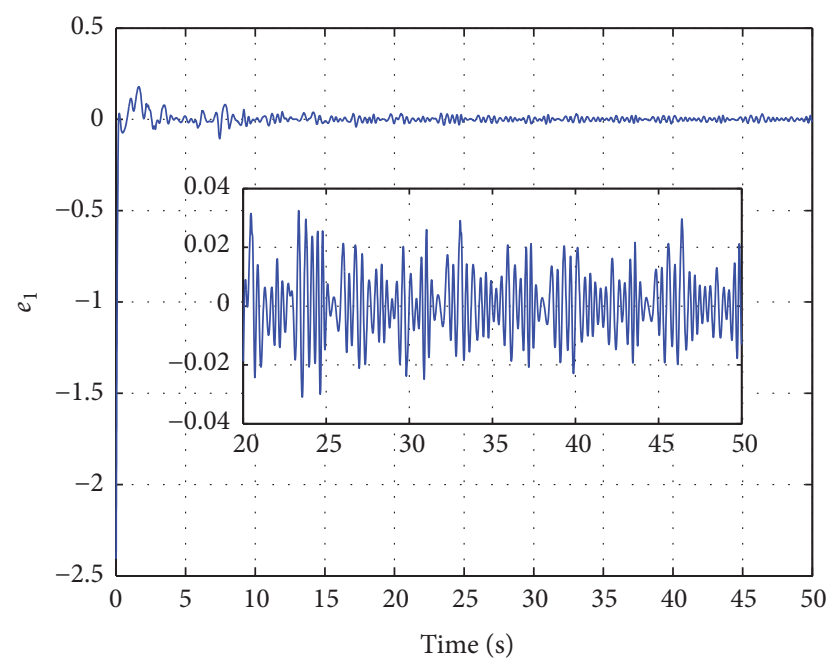

FIGURE 2: Trajectory of tracking error $e_{1}(t)$.

conditions remain the same as before. The simulation results are shown in Figures 5 and 6 , from which it can be seen that by using the method proposed in [42], the boundedness of the tracking error can be ensured, but the accurate size cannot be determined. This drawback can be overcome by employing the control scheme established in this paper.

Example 2. Consider a switched RCL circuit system [45] as shown in Figure 7, which can be described as

$$
\begin{aligned}
& \dot{\chi}_{1}=\chi_{2}+f_{1}^{\sigma(t)}\left(\bar{\chi}_{1}\right), \\
& \dot{\chi}_{2}=u(t-\tau)+f_{2}^{\sigma(t)}\left(\bar{\chi}_{2}\right)
\end{aligned}
$$

where switching signal $\sigma(t) \in\{1,2\}$ is described as Figure 4 . $f_{1}^{1}=f_{2}^{1}=(1 / L) \chi_{2}-\chi_{1}, f_{1}^{2}=-\left(1 / C_{1}\right) \chi_{1}-(R / L) \chi_{2}$, $f_{2}^{2}=-\left(1 / C_{2}\right) \chi_{1}-(R / L) \chi_{2}, \chi_{1}=q_{c}$ stands for the charge in capacitor, and $\chi_{2}=\phi_{L}$ denotes the flux in the inductance in the circuit. $C_{i}$ is the $i$ th capacitor, $L$ is the inductance, $R$ is the

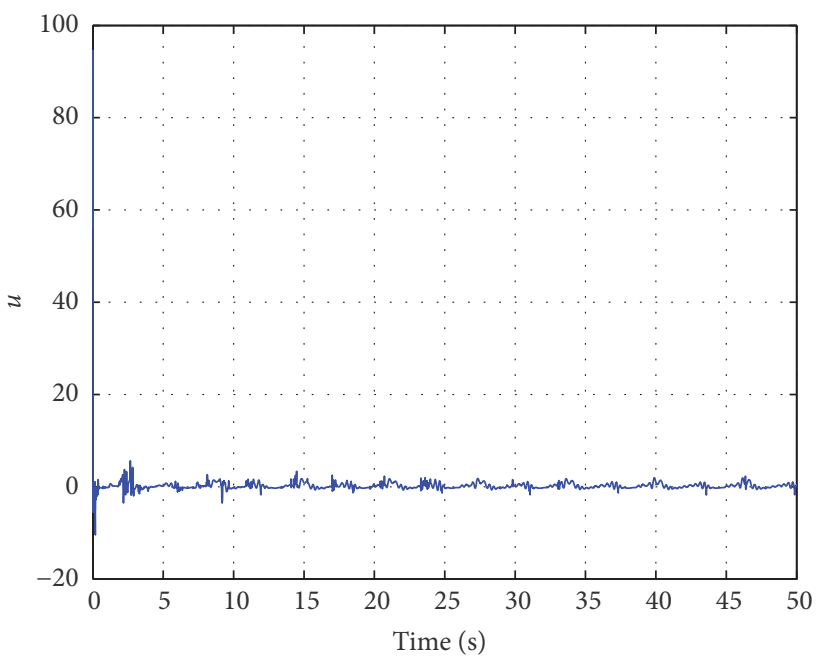

FIgURE 3: Trajectory of control input signal $u(t)$.

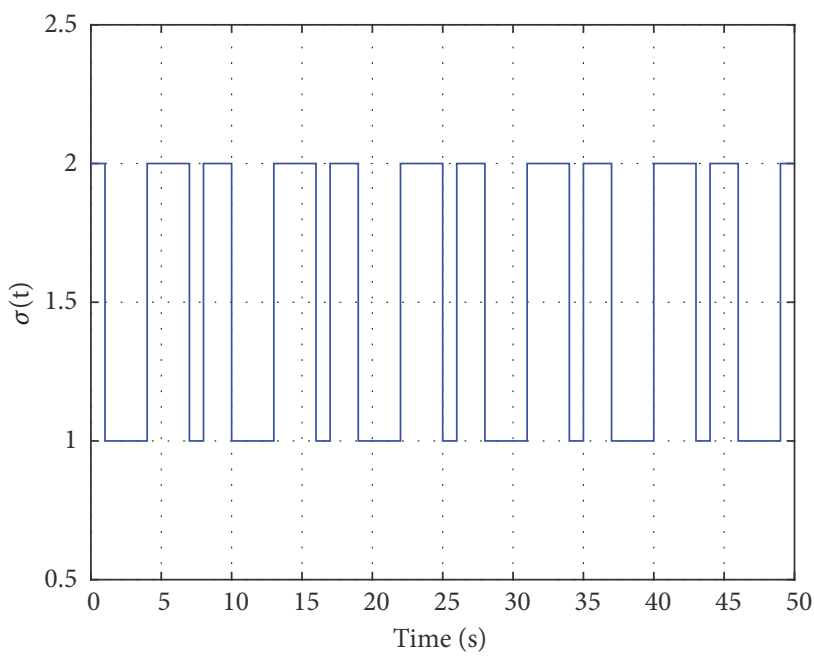

FIGURE 4: Trajectory of switching signal $\sigma(t)$.

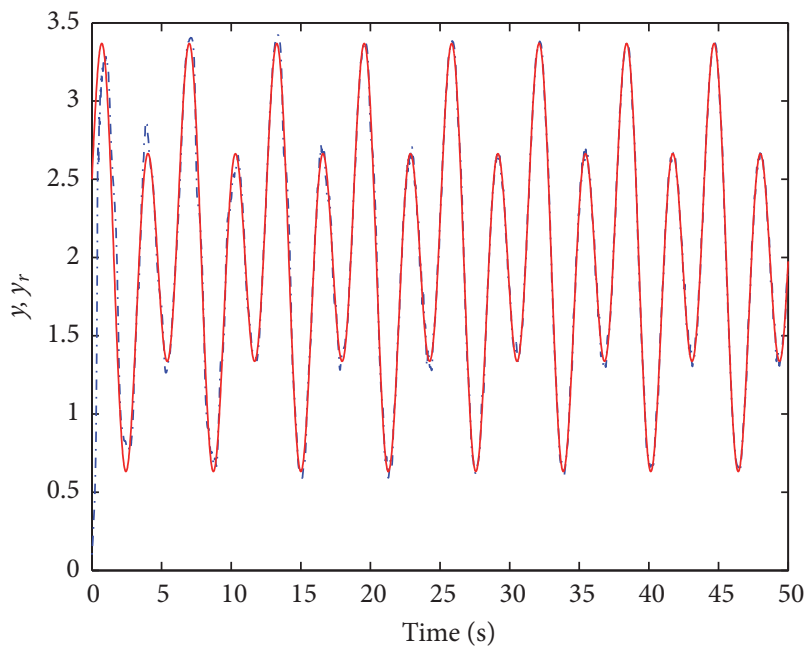

FIgURE 5: Trajectories of output signal $y(t)$ (dash-dotted line) and reference signal $y_{r}(t)$ (solid line) using method [42]. 


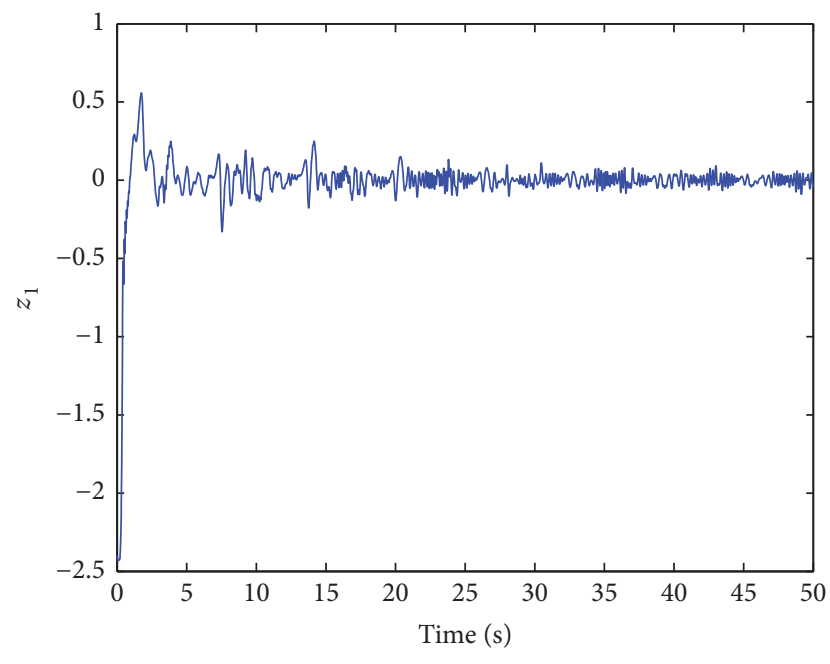

FIGURE 6: Trajectory of tracking error $e_{1}(t)$ using method [42].

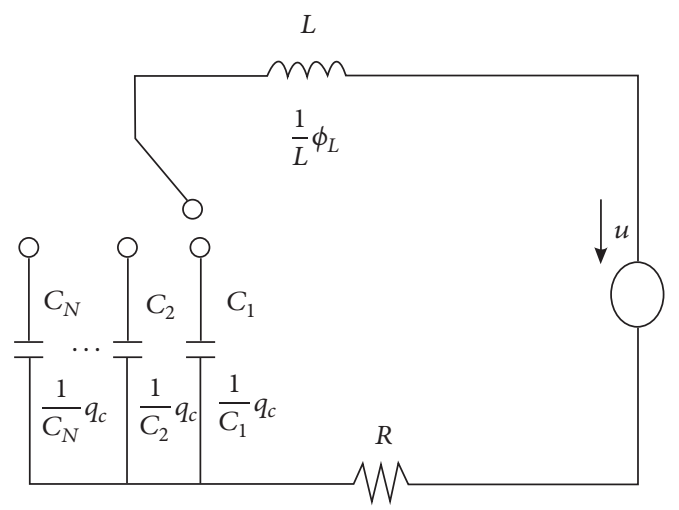

FIGURE 7: Switched RCL circuit [45].

resistance, and $u$ means the voltage. The related parameters are selected as $L=1.2, R=1, C_{1}=50$, and $C_{2}=100$. The input delay is chosen as $\tau=0.0001$. The reference signal is given as $y_{r}(t)=1.5+0.5 * \sin (t)$ and the tracking accuracy is assigned as $\epsilon=0.02$.

According to the design process of Section 3, the design parameters of the adaptive neural controllers and learning laws are chosen as $k_{1}=1.5, k_{2}=2, a_{1}=1.2, a_{2}=1.7$, $\gamma_{1}=5, \gamma_{2}=7$, and $\lambda_{1}=\lambda_{2}=10$. Two RBF NNs shown in Example 1 are employed to approximate uncertain functions in this example.

The initial conditions are given as $\chi_{1}(0)=0.1, \chi_{2}(0)=$ -0.1 , and $\widehat{\rho}_{i}=\widehat{\psi}_{i}=0, i=1,2$. The simulation results are shown in Figures 8-10, and from Figure 9, it can be seen that the tracking error achieves the given accuracy after 20 seconds.

\section{Conclusions}

This paper has addressed the adaptive tracking control problem for a class of uncertain switched systems with input delay. The main contribution of this work is that the proposed adaptive neural controller ensures the tracking error converging to

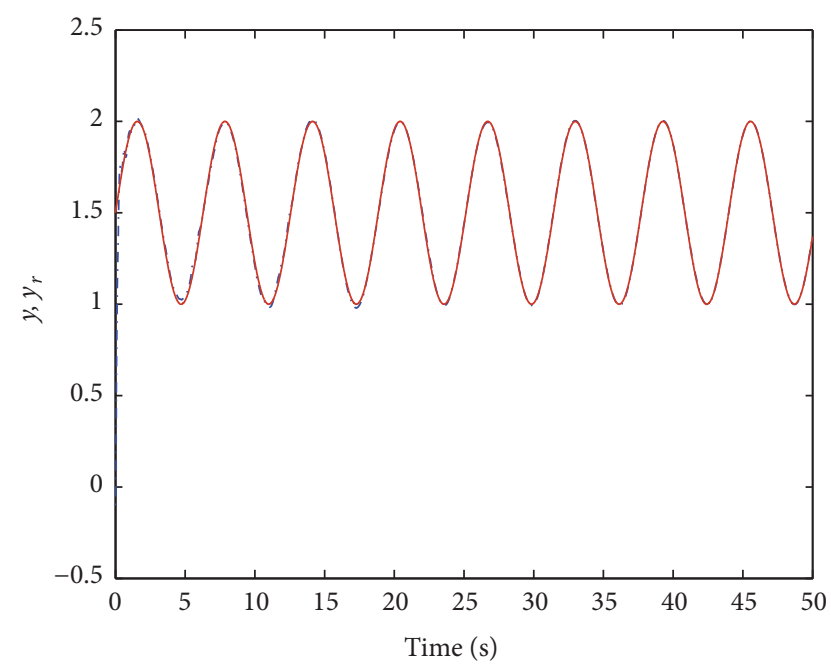

FIGURE 8: Trajectories of system output signal $y(t)$ (dash-dotted line) and reference signal $y_{r}(t)$ (solid line).

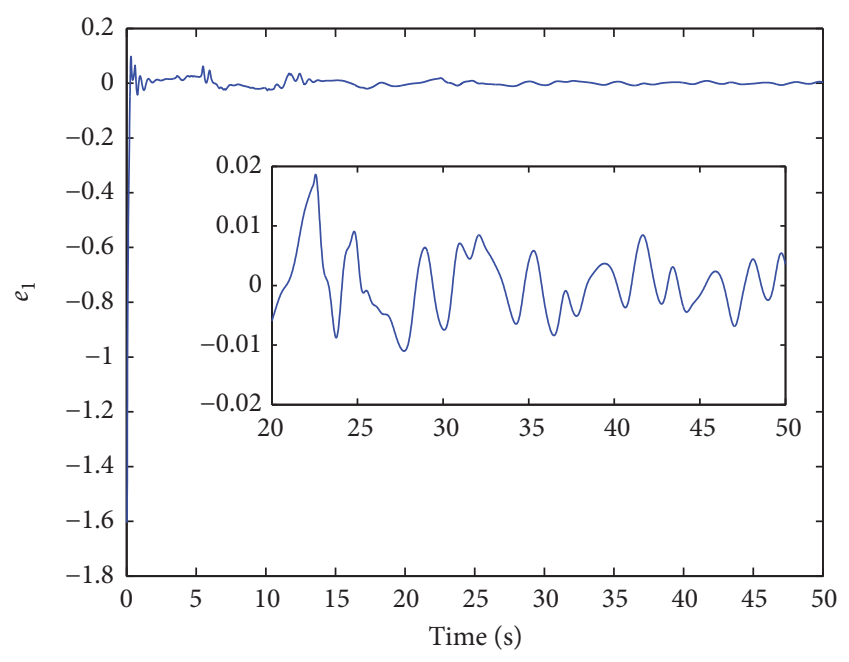

FIGURE 9: Trajectory of tracking error $e_{1}(t)$.

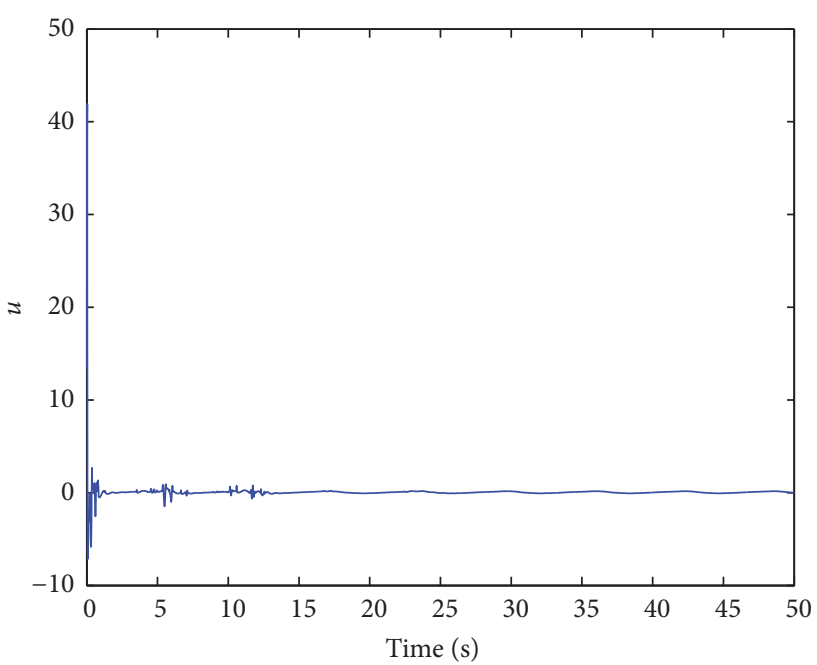

FIgURE 10: Trajectory of control input signal $u(t)$. 
the accuracy predefined a priori according to the requirement for the considered switched nonlinear system. To achieve this control performance, some nonnegative switching functions are introduced to design the desired controller. Of course, the proposed control scheme has some improvements and this topic can be further considered in the future, for example, how to develop the asymptotic tracking control scheme for the considered system (9), how to extend the proposed control method to system (9) with stochastic disturbances and nonlinearly input, etc. In addition, for the uncertain switched systems with the actuators saturation, it is a practical and challenging control problem to design an adaptive $\mathrm{NN}$ controller with the tracking accuracy known a priori.

\section{Data Availability}

This paper is a theoretical study and no data were used to support this study.

\section{Disclosure}

All authors have been personally and actively involved in substantive work leading to the report and will hold themselves jointly and individually responsible for its content.

\section{Conflicts of Interest}

The authors declare that they have no conflicts of interest.

\section{Acknowledgments}

This work is supported by the National Natural Science Foundation of China (61603003, 61703117, 61673014, 61673308, 61673339, and 61702012), the Natural Science Foundation of Anhui Province (1608085QF131), the China Postdoctoral Science Foundation (2017M620245), the Foundation of the University Research and Innovation Platform Team for Intelligent Perception and Computing of Anhui Province, the Program for Academic Top-Notch Talents of University Disciplines (gxbjZD21), and the Program for Innovative Research Team in Anqing Normal University.

\section{References}

[1] D. Liberzon and A. S. Morse, "Basic problems in stability and design of switched systems," IEEE Control Systems Magazine, vol. 19, no. 5, pp. 59-70, 1999.

[2] D. Liberzon, Switching in Cystems and Control, Birkhäuser, Boston, Mass, USA, 2003.

[3] X. Zhao, Y. Yin, L. Liu, and X. Sun, "Stability analysis and delay control for switched positive linear systems," IEEE Transactions on Automatic Control, vol. 63, no. 7, pp. 2184-2190, 2018.

[4] X. Zhao, L. Zhang, P. Shi, and M. Liu, "Stability of switched positive linear systems with average dwell time switching," Automatica, vol. 48, no. 6, pp. 1132-1137, 2012.

[5] J. Fu, R. C. Ma, and T. Y. Chai, "Global finite-time stabilization of a class of switched nonlinear systems with the powers of positive odd rational numbers," Automatica, vol. 54, pp. 360-373, 2015.
[6] J. Li, X. Yang, and J. Wu, "Adaptive tracking control approach with prespecified accuracy for uncertain nonlinearly parameterized switching systems," IEEE Access, vol. 6, no. 3, pp. 37863793, 2017.

[7] L. Long and J. Zhao, " $\mathrm{H}_{\infty}$ control of switched nonlinear systems in $p$-normal form using multiple Lyapunov functions," Institute of Electrical and Electronics Engineers Transactions on Automatic Control, vol. 57, no. 5, pp. 1285-1291, 2012.

[8] M. Jungers and J. Daafouz, "Guaranteed cost certification for discrete-time linear switched systems with a dwell time," Institute of Electrical and Electronics Engineers Transactions on Automatic Control, vol. 58, no. 3, pp. 768-772, 2013.

[9] G. Zong and H. Zhao, "Input-to-state stability of switched nonlinear delay systems based on a novel Lyapunov-Krasovskii functional method," Journal of Systems Science \& Complexity, vol. 31, no. 4, pp. 875-888, 2018.

[10] R. Ma and J. Zhao, "Backstepping design for global stabilization of switched nonlinear systems in lower triangular form under arbitrary switchings," Automatica, vol. 46, no. 11, pp. 1819-1823, 2010.

[11] N. Wang, M. J. Er, and M. Han, "Dynamic tanker steering control using generalized ellipsoidal-basis-function-based fuzzy neural networks," IEEE Transactions on Fuzzy Systems, vol. 23, no. 5, pp. 1414-1427, 2015.

[12] Y. Gao and M. J. Er, "Online adaptive fuzzy neural identification and control of a class of MIMO nonlinear systems," IEEE Transactions on Fuzzy Systems, vol. 11, no. 4, pp. 462-477, 2003.

[13] Y. Liu and Y. Li, "Adaptive fuzzy output-feedback control of uncertain SISO nonlinear systems," Nonlinear Dynamics, vol. 61, no. 4, pp. 749-761, 2010.

[14] Y. Li, K. Sun, and S. Tong, "Adaptive fuzzy robust faulttolerant optimal control for nonlinear large-scale systems," IEEE Transactions on Fuzzy Systems, vol. 26, no. 5, pp. 28992914, 2018.

[15] T. Zhang, S. S. Ge, and C. C. Hang, "Adaptive neural network control for strict-feedback nonlinear systems using backstepping design," Automatica, vol. 36, no. 12, pp. 1835-1846, 2000.

[16] C.-M. Lin and C.-F. Hsu, "Recurrent-neural-network-based adaptive-backstepping control for induction servomotors," IEEE Transactions on Industrial Electronics, vol. 52, no. 6, pp. 1677-1684, 2005.

[17] J. Li, W. Chen, J. Li, and Y. Fang, "Adaptive NN output-feedback stabilization for a class of stochastic nonlinear strict-feedback systems," ISA Transactions ${ }^{\circledR}$, vol. 48, no. 4, pp. 468-475, 2009.

[18] W. Chen and J. Li, "Globally decentralized adaptive backstepping neural network tracking control for unknown nonlinear interconnected systems," Asian Journal of Control, vol. 12, no. 1, pp. 96-102, 2010.

[19] J.-T. Huang, "Global tracking control of strict-feedback systems using neural networks," IEEE Transactions on Neural Networks and Learning Systems, vol. 23, no. 11, pp. 1714-1725, 2012.

[20] C. Hua, X. Guan, and P. Shi, "Robust output feedback tracking control for time-delay nonlinear systems using neural network," IEEE Transactions on Neural Networks and Learning Systems, vol. 18, no. 2, pp. 495-505, 2007.

[21] H. Zhang, L. Feng, and Z. Li, "A learning-based synthesis approach to the supremal nonblocking supervisor of discreteevent systems," IEEE Transactions on Automatic Control, vol. 63, no. 10, pp. 3345-3360, 2018.

[22] Y.-J. Liu and S. Tong, "Barrier Lyapunov functions for Nussbaum gain adaptive control of full state constrained nonlinear systems," Automatica, vol. 76, pp. 143-152, 2017. 
[23] C. Kwan and F. L. Lewis, "Robust backstepping control of nonlinear systems using neural networks," IEEE Transactions on Systems, Man and Cybernetics, Part A: Systems and Humans, vol. 30, no. 6, pp. 753-766, 2000.

[24] Y. Yang, G. Feng, and J. Ren, "A combined backstepping and small-gain approach to robust adaptive fuzzy control for strictfeedback nonlinear systems," IEEE Transactions on Systems, Man and Cybernetics, Part A: Systems and Humans, vol. 34, no. 3, pp. 406-420, 2004.

[25] Y. Li and S. Tong, "Adaptive fuzzy output-feedback stabilization control for a class of switched nonstrict-feedback nonlinear systems," IEEE Transactions on Cybernetics, vol. 47, no. 4, pp. 1007-1016, 2017.

[26] S. Huang and Z. Xiang, "Adaptive finite-time stabilization of a class of switched nonlinear systems using neural networks," Neurocomputing, vol. 173, pp. 2055-2061, 2016.

[27] L. Long and T. Si, "Small-gain technique-based adaptive nn control for switched pure-feedback nonlinear systems," IEEE Transactions on Cybernetics, vol. 49, no. 5, pp. 1873-1884, 2019.

[28] M. Shahvali, N. Pariz, and M. Akbariyan, "Distributed finitetime control for arbitrary switched nonlinear multi-agent systems: an observer-based approach," Nonlinear Dynamics, vol. 94, no. 3, pp. 2127-2142, 2018.

[29] M. Shahvali and J. Askari, "Cooperative adaptive neural partial tracking errors constrained control for nonlinear multi-agent systems," International Journal of Adaptive Control and Signal Processing, vol. 30, no. 7, pp. 1019-1042, 2016.

[30] Z. Zhang, C. Lin, and B. Chen, "Complete LKF approach to stabilization for linear systems with time-varying input delay," Journal of The Franklin Institute, vol. 352, no. 6, pp. 2425-2440, 2015.

[31] J. Cheng and L. L. Xiong, "Improved integral inequality approach on stabilization for continuous-time systems with time-varying input delay," Neurocomputing, vol. 160, no. 21, pp. 274-280, 2015.

[32] Y. Liu, L.-S. Hu, and P. Shi, "A novel approach on stabilization for linear systems with time-varying input delay," Applied Mathematics and Computation, vol. 218, no. 10, pp. 5937-5947, 2012.

[33] B. Zhou, Z. Lin, and G. Duan, "Truncated predictor feedback for linear systems with long time-varying input delays," Automatica, vol. 48, no. 10, pp. 2387-2399, 2012.

[34] D. Li, Y. Liu, S. Tong, C. L. Chen, and D. Li, "Neural networksbased adaptive control for nonlinear state constrained systems with input delay," IEEE Transactions on Cybernetics, pp. 1-10, 2018.

[35] H. Zhang, G. Duan, and L. Xie, "Linear quadratic regulation for linear time-varying systems with multiple input delays," Automatica, vol. 42, no. 9, pp. 1465-1476, 2006.

[36] B. Chen, X.-P. Liu, S.-C. Tong, and C. Lin, "Observer-based stabilization of T-S fuzzy systems with input delay," IEEE Transactions on Fuzzy Systems, vol. 16, no. 3, pp. 652-663, 2008.

[37] Y.-E. Wang, X.-M. Sun, Z. Wang, and J. Zhao, "Construction of Lyapunov-Krasovskii functionals for switched nonlinear systems with input delay," Automatica, vol. 50, no. 4, pp. 12491253, 2014.

[38] B. Niu and L. Li, "Adaptive backstepping-based neural tracking control for MiMo nonlinear switched systems subject to input delays," IEEE Transactions on Neural Networks and Learning Systems, vol. 29, no. 6, pp. 2638-2644, 2018.

[39] K. Krstic, I. Kanellakopouls, and P. V. Kokovic, Nonlinear and Adaptive Control Design, Wiley, New York, NY, USA, 1995.
[40] W. Chen, S. S. Ge, J. Wu, and M. Gong, "Globally stable adaptive backstepping neural network control for uncertain strict-feedback systems with tracking accuracy known a priori," IEEE Transactions on Neural Networks and Learning Systems, vol. 26, no. 9, pp. 1842-1854, 2015.

[41] J. J. Slotine and W. Li, Applied Nonlinear Control, Prentice Hall, Englewood Cliffs, 1991.

[42] X. Zhao, X. Zheng, B. Niu, and L. Liu, "Adaptive tracking control for a class of uncertain switched nonlinear systems," Automatica, vol. 52, pp. 185-191, 2015.

[43] G. Lai, Z. Liu, Y. Zhang, C. L. Philip Chen, and S. Xie, "Adaptive backstepping-based tracking control of a class of uncertain switched nonlinear systems," Automatica, vol. 91, pp. 301-310, 2018.

[44] Y. Li, S. Sui, and S. Tong, "Adaptive fuzzy control design for stochastic nonlinear switched systems with arbitrary switchings and unmodeled dynamics," IEEE Transactions on Cybernetics, vol. 47, no. 2, pp. 403-414, 2017.

[45] L. Liu, Y. Liu, and S. Tong, "Neural networks-based adaptive finite-time fault-tolerant control for a class of strict-feedback switched nonlinear systems," IEEE Transactions on Cybernetics, vol. 49, no. 7, pp. 2536-2545, 2019. 


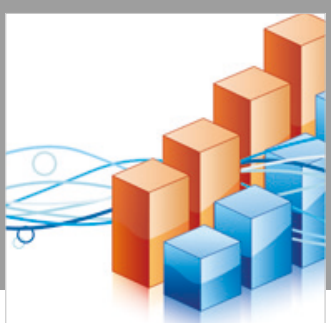

Advances in

Operations Research

\section{-n-m}
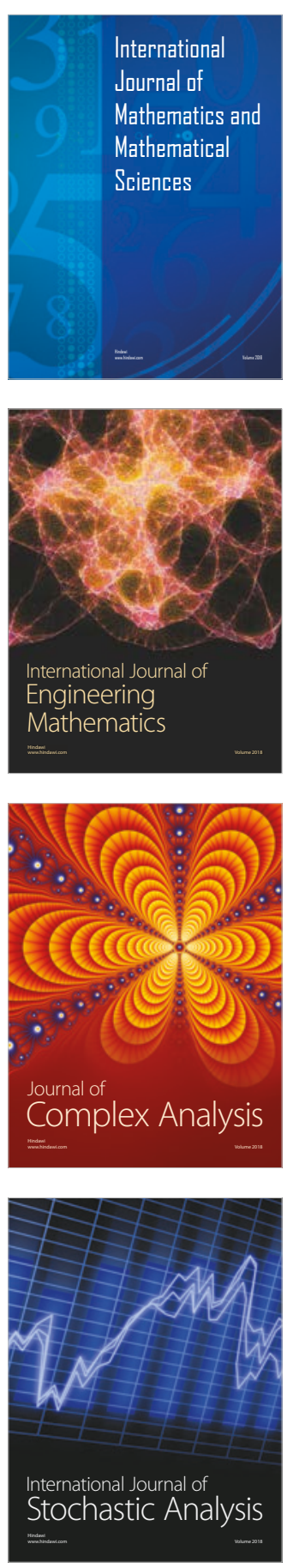
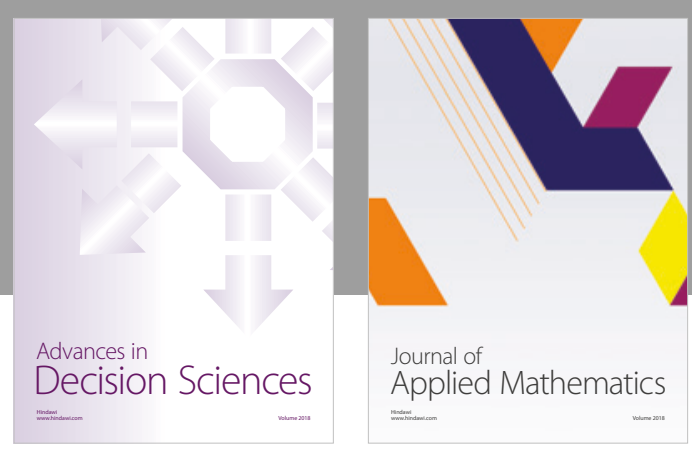

Journal of

Applied Mathematics
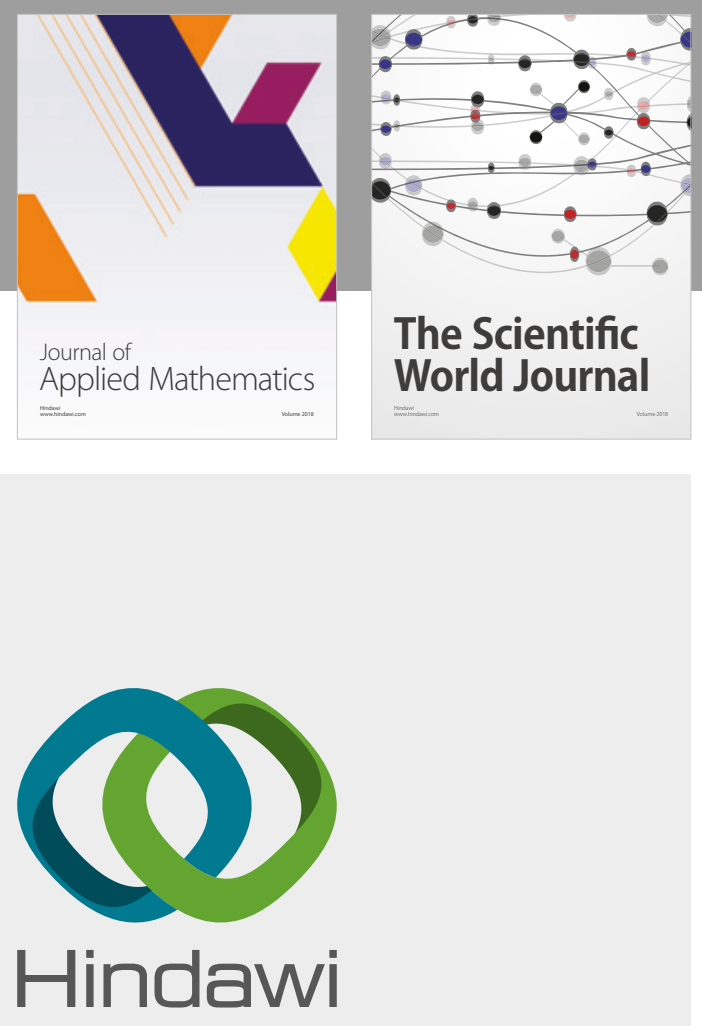

Submit your manuscripts at

www.hindawi.com

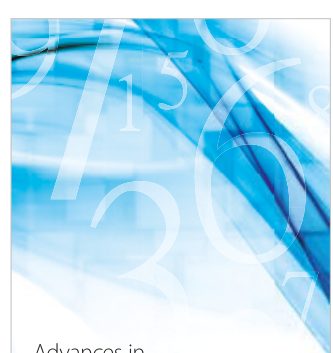

Advances in
Numerical Analysis
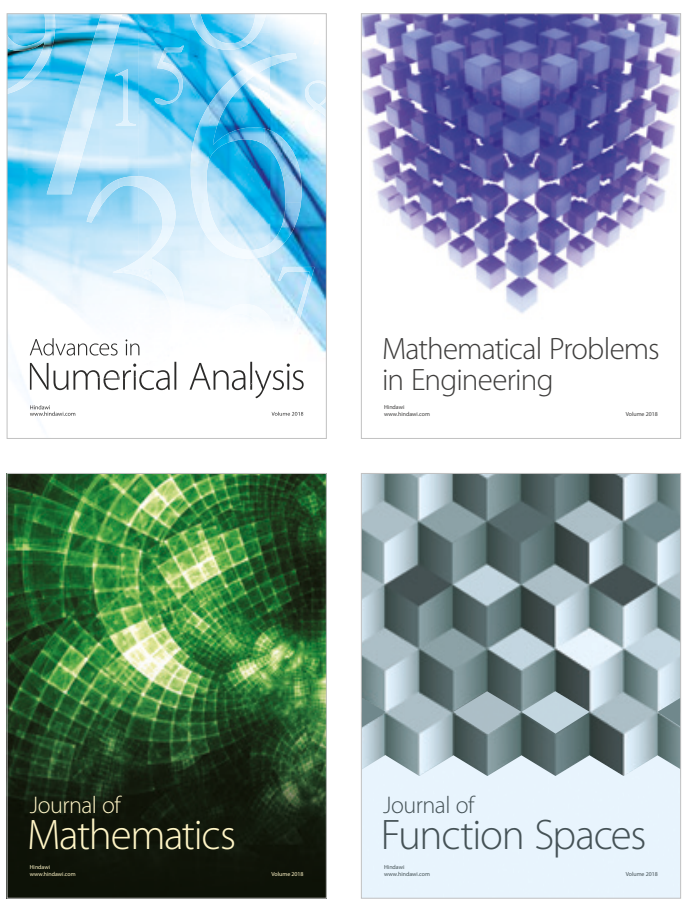

Mathematical Problems in Engineering

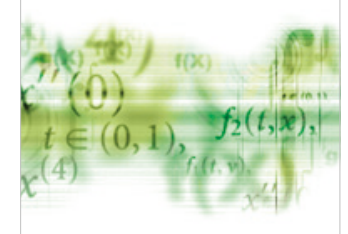

International Journal of

Differential Equations

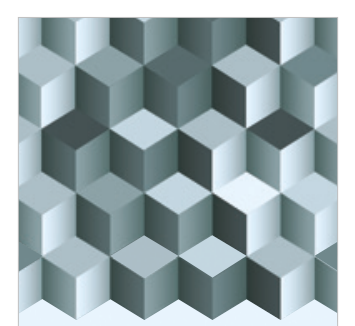

Journal of

Function Spaces
The Scientific

World Journal

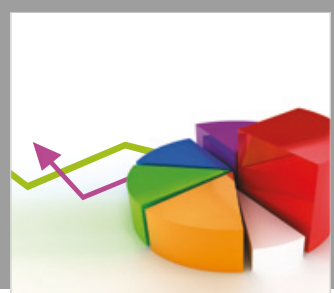

Journal of

Probability and Statistics
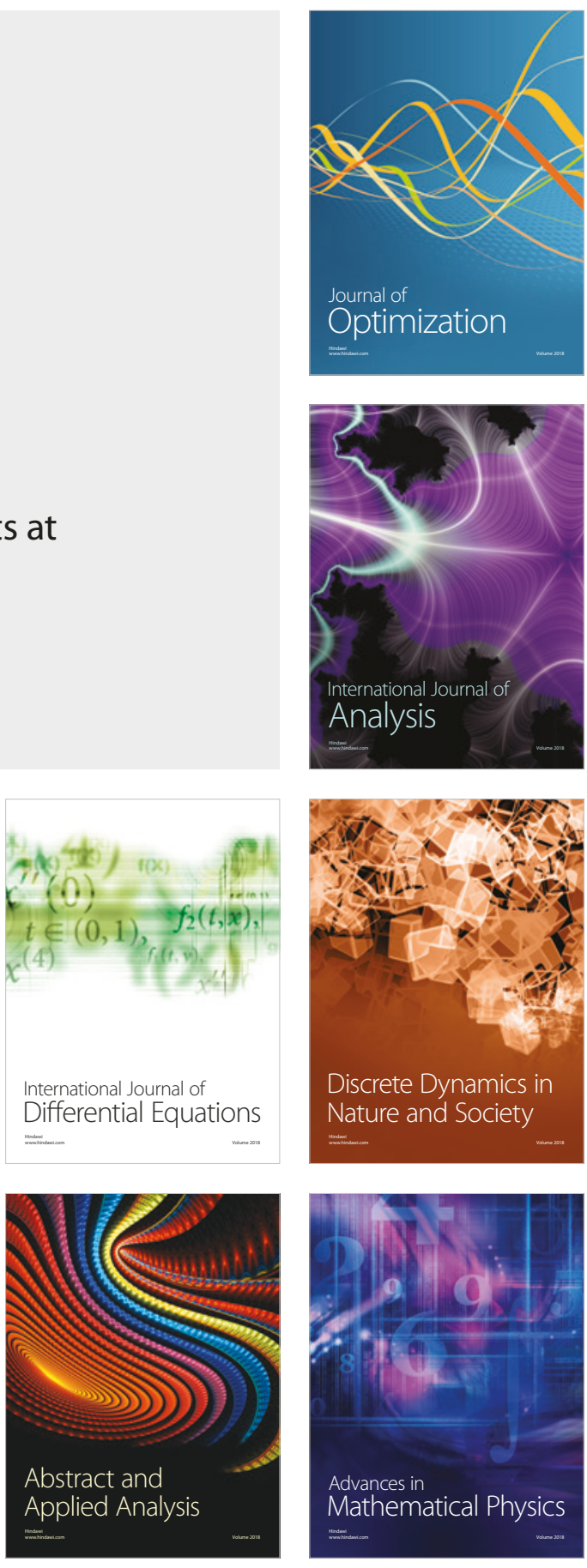